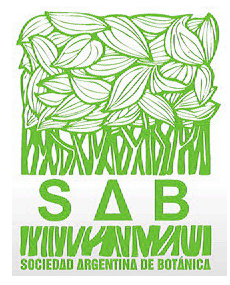

\title{
Caracterización y estado de conservación de la VEGETACIÓN RIBEREÑA DE LA CUENCA MEDIA DEL RÍO Cuarto (Córdoba, Argentina)
}

\author{
CHARACTERIZATION AND CONSERVATION STATUS OF THE RIPARIAN \\ VEGETATION OF THE MIDDLE BASIN OF THE RÍo CuARTo (CóRdobA, \\ Argentina)
Evangelina Natale ${ }^{1,2,3 *}(\mathbb{D})$, Marcelo Arana ${ }^{1}$ D, Gabriela Villalba ${ }^{\dagger}$, Herminda Reinoso ${ }^{1}$ (D), Marina de la Reta ${ }^{1,3}$ (D) Antonia Oggero' ${ }^{1}$ (D)

\author{
1. Dpto Ciencias Naturales, \\ Universidad Nacional de Río \\ Cuarto. \\ 2. CONICET. \\ 3. Fundación Conservación y \\ Desarrollo (ConyDes). \\ *enatale@exa.unrc.edu.ar; \\ evangenatale@yahoo.com.ar
}

Citar este artículo NATALE, E., M. ARANA, G. VILLALBA,$+ H$. REINOSO, M.DE LA RETA \& A. OGGERO. 2019. Caracterización y estado de conservación de la vegetación ribereña de la cuenca media del río Cuarto (Córdoba, Argentina). Bol. Soc. Argent. Bot. 54: 105-123.

DOI: http://dx.doi. org/10.31055/1851.2372.v54. n1.23589

Recibido: 16 Agosto 2018

Aceptado: 21 Noviembre 2018

Publicado: 28 Marzo 2019

Editor: Guillermo Funes (iD)

ISSN versión impresa 0373-580X

ISSN versión on-line 1851-2372

\section{SUMMARY}

Background and aims: The processes of change of land use have had a very profound impact on the ecological patterns and processes of landscapes in recent times, owing to that, this study aims to characterize the vegetation patches associated with the middle basin of the río Cuarto and to determine its conservation status in order to delineate management strategies that allow planners to choose the best alternative for urban development.

M\&M: Vegetation censuses were carried out in the different environments. The environmental units were digitized and an Integral Environmental Evaluation Index included in a Geographic Information System platform was applied to the define the conservation value.

Results: Seven vegetation units belonging to the biogeographic district of Espinal were delimited and 340 remnants of natural vegetation were digitized. According to the Integral Environmental Evaluation Index, more than $50 \%$ of the UA are in bad status of conservation, where the patches with regular and good status presented a high value of ecological functionality within the basin. By other hand, less than $1 \%$ of the environmental units are in very good status of conservation.

Conclusions: These results allowed us to generate management recommendations in order to promote its preservation and improvement of its environmental health. These recommendations could be included in the territorial planning of the cities associated to the studied basin.

\section{KEY WORDS}

Biodiversity, conservation, ecosystem services, landscape metrics, territorial planning.

\section{RESUMEN}

Introducción y objetivos: Los procesos de cambio de uso de la tierra han tenido un impacto muy profundo en los patrones y procesos ecológicos de los paisajes en los últimos tiempos, por lo que éste trabajo tiene por objetivos caracterizar los parches de vegetación asociados a la cuenca media del río Cuarto y determinar su estado de caracterización, con el fin de delinear estrategias de manejo que permitan a los planificadores optar por la mejor alternativa para el desarrollo urbano.

M\&M: Se realizaron censos de vegetación en los diferentes ambientes y se digitalizaron las unidades ambientales; luego se les calculó el Índice Integral de Evaluación Ambiental, incluido en una plataforma de Sistema de Información Geográfica, para definir el valor de conservación.

Resultados: Se definieron siete unidades de vegetación pertenecientes al distrito biogeográfico Espinal y se digitalizaron 340 parches. El índice integral de evaluación ambiental arrojó que más del $50 \%$ de las Unidades Ambientales se encontraron en mal estado de conservación, donde los parches regulares y buenos presentaron un alto valor de funcionalidad ecológica dentro de la cuenca. Por otro lado, menos del $1 \%$ de la unidades ambientales entraron en la categoría de muy buen estado de conservación.

Conclusiones: Estos resultados permitieron generar una serie de recomendaciones de manejo tendientes a su preservación y mejoramiento de su salud ambiental que pueden ser incluidas en el ordenamiento territorial de las ciudades asociadas a la cuenca en estudio.

\section{Palabras clave}

Biodiversidad, conservación, métricas del paisaje, ordenamiento territorial, servicios ecosistémicos. 


\section{INTRODUCCIÓN}

Los procesos de cambio de uso de la tierra son una expresión de las transformaciones socioeconómicas que han tenido un impacto muy profundo en los patrones y procesos ecológicos de los paisajes en los últimos tiempos (Costanza et al., 1997; Parcerisas et al., 2012). Así, se ha reconocido que la expansión de las urbes junto a las prácticas agrícolas se configuran como una de las principales causas antrópicas que ejercen una presión negativa significativa sobre los recursos naturales (Jaeger et al., 2010). Esta transformación del paisaje subyace a la mayoría de los problemas de conservación ya que el impacto de las actividades antrópicas ha llevado a la pérdida de servicios ecosistémicos, como la capacidad de captación de agua, la protección de suelos, la regulación de los ciclos de nutrientes y la reducción de los efectos del cambio climático global (ej: prevención de inundaciones y atenuación de las consecuencias de las sequías) (Szpeiner et al., 2007; Bilenca et al., 2008). A esto se le suma la declinación de las poblaciones de especies nativas, algunas hasta la extinción (Benton et al., 2003; Dirzo \& Raven, 2003). Es por ello que, en el debate internacional sobre el Cambio Climático, el concepto de servicios ecosistémicos se ha convertido en un tema central y se ha concluido que el progresivo aumento de la demanda de los recursos naturales, y la consecuente pérdida de los servicios que proveen, hace que la protección de los ecosistemas y la biodiversidad se conviertan en una cuestión esencial (Frank et al., 2012).

En este devenir se deja de tener en cuenta que el ambiente urbano (ciudades, suburbios, pueblos, etc.) y los sistemas productivos dependen de los ecosistemas, naturales o seminaturales, que se encuentran en su interior o en la periferia, para la provisión de las funciones y servicios mencionados anteriormente (Li et al., 2005). Desde esta perspectiva, los remanentes de vegetación inmersos en matrices agrícolas-urbanas tienen un valor económico y social en el mantenimiento de la salud pública y el bienestar, así como en la contribución a la conservación, soportando una biodiversidad única (Fuller et al., 2007; Lawson et al., 2008; Escobedo et al., 2011). La mitigación de los impactos del crecimiento de las ciudades y los sistemas productivos sobre los sistemas y procesos naturales es complicada, debido al alto valor económico que poseen las tierras, a la diversidad de partes interesadas y a una falta de práctica de investigación en relación con la amenaza de la urbanización a la diversidad biológica (Gordon et al., 2009). Por lo tanto, las matrices compuestas por agro-ecosistemas, urbanizaciones y remanentes naturales o seminaturales proveen la oportunidad de fusionar el manejo ecológico con el diseño del paisaje para brindar una variedad de servicios y bienes sociales (Williams et al., 2009), a través de la generación de planes de gestión y políticas que permitan reducir los efectos negativos de las actividades antrópicas (Gallardo et al., 2011).

Frente a esta realidad, la gestión ambiental se enfrenta con la necesidad de transferir el concepto de servicios ecosistémicos a los procesos de ordenamiento territorial (de Groot, 2006; Burkhard et al., 2010), y de manejar paisajes enteros, que a menudo consisten en complejos mosaicos de diferentes parches de hábitat y ecosistemas que interactúan entre sí (Lindenmayer et al., 2008). Por tal motivo, las evaluaciones de paisaje se configuran como una herramienta útil y rápida, con enfoques integrados y de fácil aplicación, que permiten la cuantificación de los beneficios derivados de los ecosistemas para que los planificadores y políticos puedan incluirlas en el desarrollo de sus planificaciones territoriales (de Groot, 2006; Sandstrom et al., 2006; Cadenasso et al., 2007; Burkhard et al., 2010; Farley \& Costanza, 2010; Parcerisas et al., 2012).

Un enfoque, muy utilizado, para evaluar la estructura de los patrones espaciales relacionados con el paisaje y el impacto en los servicios ecosistémicos es el uso de métricas del paisaje (MP) (Frank et al., 2012). Estas métricas son útiles para la aplicación de los conceptos de la ecología del paisaje a la planificación sostenible (Lin et al., 2007) y al monitoreo de los procesos de desarrollo (Uuemaa et al., 2009). Una vasta literatura avala el punto de vista de que, para la conservación efectiva a escala de paisaje, no solo se requiere de información sobre la extensión y/o superficie de los remanentes sino también información sobre el arreglo espacial, la composición y el estado la vegetación natural; siendo, este último factor, dependiente de las modificaciones antrópicas, el grado de conectividad, la intensidad de uso del suelo, entre otros (Forman, 1995; Primack \& Ros, 2002; Natale et al., 2015). En este contexto, los 


\section{E. Natale et al. - Caracterización ambiental de la cuenca del río Cuarto}

mapas de vegetación y ambientes se configuran como instrumentos básicos para el conocimiento de un área geográfica, ya que resumen propiedades de los ecosistemas presentes y, en muchos casos, permiten inferir aspectos funcionales de los paisajes constituyéndose en insumos necesarios para los planes de gestión y ordenamiento (Kandus et al, 2006).

Además, la incorporación de este tipo de variables en índices globales o integrales y en modelos que permitan obtener resultados espaciales explícitos, encuadrados dentro de un entorno de Sistema de Información Geográfica, se vuelven especialmente útiles (Branquart et al., 2008; Van der Biest et al., 2014). Hasta la fecha, sólo unos pocos estudios han combinado, en un enfoque intersectorial, los conceptos de servicios ecosistémicos y las métricas del paisaje en las aplicaciones prácticas dentro de la planificación territorial (Lin et al., 2007; Frank et al., 2012). Se puede citar el trabajo realizado por Natale et al. (2015) quienes propusieron un índice, que incorpora métricas de paisaje en un contexto SIG, para evaluar la integridad ecológica de remanentes de vegetación natural y seminatural, inmersos en un medio urbano donde los resultados fueron incorporados en el plan de ordenamiento de dicha ciudad.

En nuestro país la desaparición de los sistemas naturales y semi-naturales fue y continúa siendo a expensas de la expansión de la frontera agrícola y del desarrollo urbano (Grau et al., 2005; Donald \& Evan, 2006). Para el caso particular de los bosques, que a comienzos del siglo XX cubrían casi el $40 \%$ del continente sudamericano, actualmente no llegan al 12\% (UMSEF, 2015). Stocker et al. (2013) alertaron, para el 2014, que el 4,3\% de la deforestación global ocurría en la Argentina. En la provincia de Córdoba, de las 1653210 hectáreas de superficie total, los bosques nativos originales representaban alrededor de 71,4\% (12000000 ha) mientras que, ya para el año 2008, sólo ocupaban menos del 3,6\% (594000 ha) (Zak et al., 2008). Particularmente, son destacables las actividades de deforestación y degradación que han sufrido los bosques de ribera, uno de los ecosistemas más importantes en cuanto a los servicios ecosistémicos que brindan, ya que cumplen funciones como la estabilización de las márgenes, captación y regulación del ciclo del agua, regulación de los ciclos de inundación y de nutrientes, construcción y asimilación del suelo, neutralización de los desechos tóxicos, y corredores para la fauna y la flora, entre otros (Elosegi \& Sabater, 2009; Romero et al., 2014).

En este contexto se enmarca el paisaje de la cuenca media del río Cuarto, ubicada al sur de la provincia de Córdoba, en la región central de Argentina, que presenta un número considerable de relictos de vegetación natural y seminatural asociados a las márgenes de sus ríos y arroyos, distribuidos en forma de parches y aislados entre sí por una matriz constituida por agro-ecosistemas y áreas urbanas. Tal como lo informa Elosegi \& Sabater (2009) en otros paisajes similares, las actividades antrópicas que allí ocurren (extracción de áridos, basurales a cielo abierto, urbanizaciones, agricultura, entre otras) están llevando a la conversión de éstos sistemas naturales modificando las características físicas y químicas, tanto del cauce como de la calidad del agua, e influyendo fuertemente en la biota, en el funcionamiento del sistema fluvial y en la salud humana de las comunidades asociada a la cuenca.

Por lo expuesto, los objetivos de este trabajo fueron realizar una caracterización de la vegetación de la cuenca media del río Cuarto, diagnosticar el estado de conservación de los remanentes de bosque ribereño, a través del ajuste metodológico del Índice Integral de Evaluación Ambiental desarrollado por Natale et al. (2015) y evaluar el riesgo que posee cada remanente, asociado a los potenciales impactos que generan las actividades antrópicas que se desarrollan en el área. Todo ello con la finalidad de generar recomendaciones de manejo que sean de utilidad en la planificación del desarrollo urbano de las localidades asociadas al área de estudio.

\section{Materiales y Métodos}

\section{Área de estudio}

El área de estudio se encuentra en la cuenca media del río Cuarto que comienza en la confluencia del río las Tapias y Piedras Blancas (32 53 '54,83'S $64^{\circ} 44^{\prime} 10,01^{\prime \prime}$ 'O a $650 \mathrm{msnm}$ ), donde el curso de agua se denomina Chocancharava, hasta la localidad de

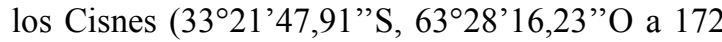
msnm) (Fig. 1). Dicha zona se encuentra inmersa en el distrito biogeográfico del Espinal, perteneciente a 


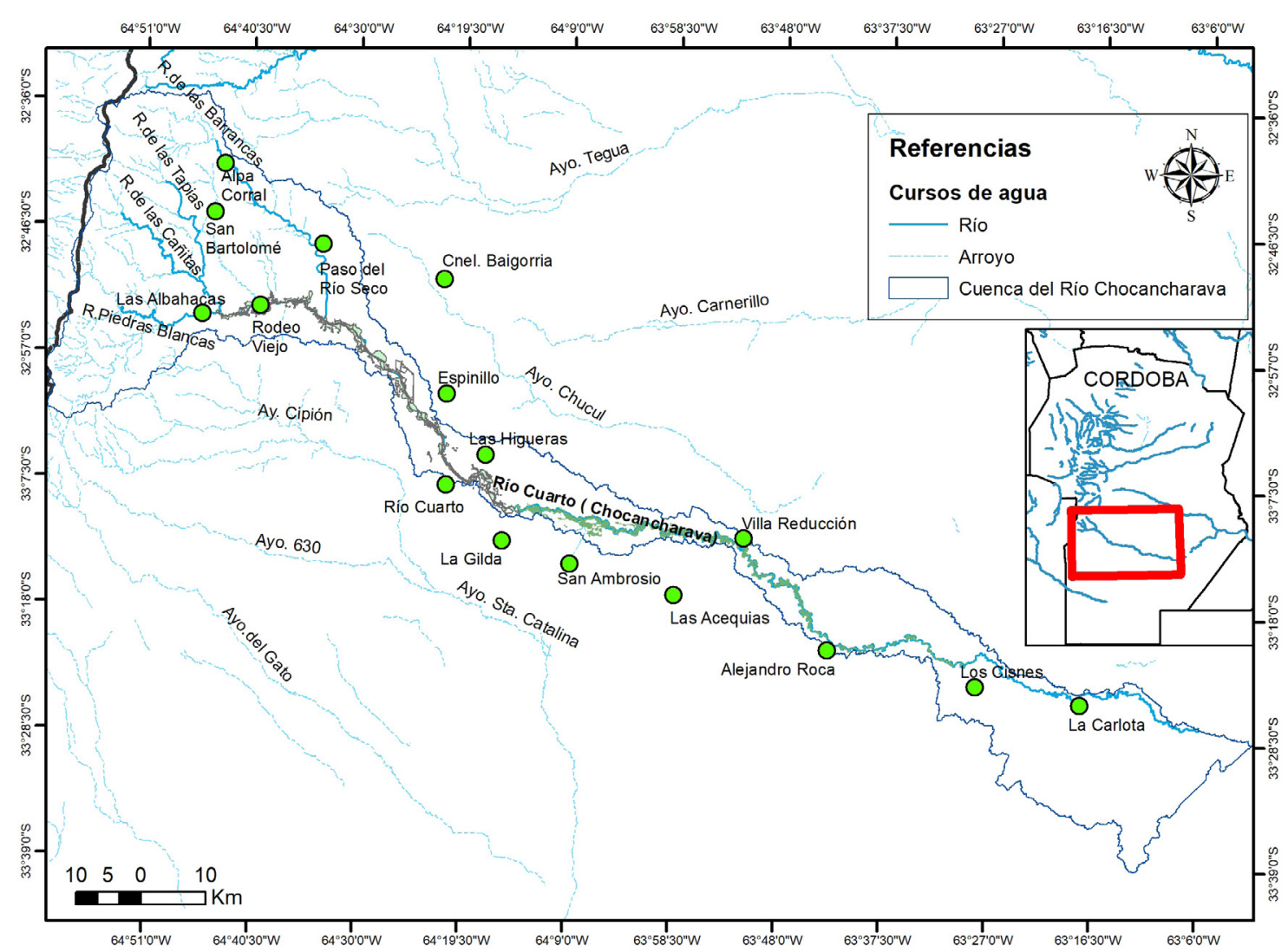

Fig. 1. Ubicación geográfica de la Cuenca media del río Cuarto (río Chocancharava).

la provincia biogeográfica Pampeana (Arana et al. 2017). Este distrito se caracteriza por la presencia de bosque xerófilo abierto, cuyos elementos arbóreos característicos son: Prosopis alba Griseb. (algarrobo blanco), Aspidosperma quebrachoblanco Schltdl. (quebracho blanco), Schinus fasciculatus (Griseb.) I.M. Johnst. (moradillo), Schinus longifolius (Lindl.) Speg. (moradillo), Celtis ehrembergiana (Klotzsch) Liebm. (tala), Geoffroea decorticans (Gillies ex Hook. \& Arn.) Burkart (chañar) y Jodina rhombifolia (Hook. \& Arn.) Reissek (peje), acompañados en menor medida por Vachellia caven (Molina) Seigler \& Ebinger (espinillo) y Porlieria microphylla (Baill.) Descole, O’Donell \& Lourteig (cucharero). Se pueden encontrar, de manera intercalada, poblaciones de Trithrinax campestris (Burmeist.) Drude \& Griseb. (palma). Hacia el sudoeste aparece Prosopis caldenia Burkart (caldén), árbol endémico representativo de la Provincia biogeográfica
Pampeana por antonomasia, acompañado de los arbustos Baccharis articulata (Lam.) Pers. (carqueja) y Lippia turbinata Griseb. (poleo). Predominan especies de cactáceas pertenecientes a los géneros Opuntia Mill. (tunas) y Cereus Mill. (cardones). En el estrato herbáceo se destacan las Poaceae pertenecientes a los géneros Nassella Desv. (flechillas) y Setaria Pal. Beauv. (cola de zorro). En zonas de mayor humedad, a lo largo de los ríos aparecen Salix humboldtiana Willd. var. humboldtiana (sauce criollo), Sambucus australis Cham. \& Schltdl. (saúco), Baccharis salicifolia (Ruiz \& Pav.) Pers. (chilca) entre otras (Arana \& Bianco, 2011).

\section{Método}

Para la caracterización de la vegetación se efectuaron censos de vegetación en donde el tamaño de las unidades de muestreo se determinó por el método de área mínima con la corrección 


\section{E. Natale et al. - Caracterización ambiental de la cuenca del río Cuarto}

propuesta por Cain (1938). Se realizaron censos distribuidos al azar a lo largo del área de estudio, en donde se registró la presencia y cobertura-abundancia por especie utilizando la escala de Braun Blanquet (1979). Se recolectó y herborizó el material y se confeccionó la lista de los taxones registrados, indicando la unidad de vegetación donde se encontraban, los endemismos específicos y las entidades exóticas. Todo el material herborizado se determinó en laboratorio y los ejemplares de referencia se depositaron en el herbario RCVC (Thiers, 2018). Además, para delimitar las comunidades vegetales se llevaron a cabo análisis fitosociológicos a través del método de agrupamiento con el programa PC-ord.

La cartografía de base se construyó mediante la utilización de imágenes satelitales obtenidas del Google Earth Pro 7.3 (2017) con tamaño pixel $0,5 \mathrm{~m}^{2}$ donde se identificaron los diferentes componentes ambientales de la cuenca media del río Cuarto (ríos, arroyos, vías de comunicación, zonas urbanas, zonas rurales, etc.). A partir de técnicas de clasificación visual y verificación a campo, dentro del área definida por la línea de ribera geológica que delimita la zona inundable en crecidas de gran magnitud (Fernández et al., 2009), se definieron las unidades ambientales utilizando el criterio fisonómico estructural (vegetación) definido por Gómez Orea \& Gómez Villarino (2013). De acuerdo con estos autores, se entiende por unidades ambientales a recintos territoriales relativamente homogéneos que representan externamente el ecosistema subyacente; permite por tanto hacer una aproximación por sistemas al conocimiento del medio físico y permite un entendimiento más real, útil y operativo del mismo; por eso se les llama también unidades de integración o de síntesis. Una vez obtenido el mapa de unidades ambientales se procedió a calcular el índice integral de evaluación ambiental (IIEA) propuesto por Natale et al. (2015). Dicho índice posee tres componentes: 1) la integridad ecológica conformada por las métricas del paisaje: tamaño, forma y tipo de parche, tipo de contacto y tipo de borde; 2) la importancia geológica dado por un valor de protección y un valor de singularidad; 3 ) variables de contexto referentes al impacto antrópico que incluyeron la presencia de caminos, presencia de asentamientos, porcentaje de superficie raleada y/o desmontada y porcentaje de superficie invadida por especies exóticas. Las variables definidas en cada uno de estos componentes se integran en una ecuación general que, mediante algoritmos matemáticos, intentan reflejar la interacción de las mismas. Donde la ecuación muestra una relación de tipo limitante entre los tres componentes, por lo que un parche debe presentar valores aceptables de los tres para encontrarse en un estado ambiental adecuado. Finalmente, se le agrega un valor adicional a las unidades ambientales, que por su forma y ubicación espacial, pueden ser consideradas corredores biológicos (Anexo I). Debido a que el índice presentado por Natale et al. (2015) utilizó variables del componente geológico, específicas a una situación geográfica y ambiental particular, en este trabajo se redefinieron a partir de estudios de base geológicos realizados en la zona (Sagripanti et al., 2005).

Finalmente, para la evaluación del riesgo, de cada unidad ambiental, asociado a los diferentes usos antrópicos se realizó un análisis de componentes principales categórico utilizando el programa IBM SPSS Statistics 20. En dicho análisis se utilizaron todas las variables, incluidas en el índice Integral de Evaluación Ambiental (Anexo I), las cuales fueron recodificadas como variables categóricas; y se consideró la totalidad de las unidades ambientales identificadas previamente como casos.

\section{Resultados}

Caracterización de la vegetación de los parches de la cuenca media del río Chocancharava.

El relevamiento arrojó que existen un total de 8795,6 ha de vegetación ribereña que ocupan un $2,9 \%$ de la superficie total de la cuenca del río Cuarto, tomando desde la desembocadura de los ríos y arroyos de cuenca alta hasta la localidad de los Cisnes, a partir de donde comienza cuenca baja (Fig. 1). Esta superficie se presentó como fragmentos aislados con un grado importante de alteración, delimitando un total de 340 parches, con escasa conexión entre ellos. Estos ambientes pertenecen al bosque ribereño de la provincia biogeográfica Pampeana (Arana et al., 2017) que, a lo largo de la trayectoria del río, aparecen de manera intercalada formaciones de estratos boscosos y arbustivos en distintos estados de sucesión ecológica. En ocasiones, estos bosques se encuentran reemplazadas por formaciones boscosas de especies exóticas invasoras como Ligustrum 
lucidum Ait. (siempre verde), Melia azedarach L. (paraíso), Gleditsia triacanthos L. (acacia negra), Ulmus pumila L. (olmo), Broussonetia papyrifera (L.) Vent. (morera de papel), Arundo donax L. (caña de Castilla) y Salix fragilis L. (sauce frágil). Según su fisonomía, estructura y composición se definieron las siguientes unidades de vegetación (Fig. 2-4):

Bosque: sectores con predominio de ejemplares leñosos xerófilos o semi xerófilos. Las variantes definidas fueron:

Bosque pampeano primario: El estrato de leñosas posee mayor altura y cobertura, llegando aproximadamente hasta los 7-8m y caracterizado por la presencia de Prosopis alba (algarrobo), Aspidosperma quebrachoblanco (quebracho blanco), Vachellia caven (espinillo), Celtis ehrenbergiana (tala), Schinus fasciculatus (moradillo), Prosopis caldenia (caldén), Geoffroea decorticans (chañar) y Jodina rhombifolia (peje). Acompañados, en el estrato arbustivo, por Sida rhombifolia L., Porlieria microphylla (cucharero), Solanum pseudocapsicum L., Ephedra triandra Tul. emend. J.H. Hunz (pico de loro), Cestrum parqui L'Herit. (duraznillo negro), Lepechinia floribunda (Benth.) Epling y Baccharis articulata (carqueja). En el estrato herbáceo aparecieron frecuentemente Solanum sisymbrifolium Lam., Cantinoa mutabilis (Rich.) Harley \& J.F.B. Pastore, Selaginella sellowii Hieron., Nassella neesiana (Trin. \& Rupr.) Barkworth y Passiflora caerulea L. (pasionaria), entre otros. En el área solo estuvo representado por dos parches que sumaron tan sólo 97,29 ha, el $1,1 \%$ de la superficie total del área estudiada. Es importante destacar que esta superficie presenta la distribución más austral de Aspidosperma quebracho-blanco.

Bosque pampeano secundario: Caracterizado por un estrato de leñosas con ausencia de algarrobos y caldenes y una altura de hasta $2-3 \mathrm{~m}$, en el cual se observaron, como especies más comunes, a Vachellia caven (espinillo), Celtis ehrenbergiana (tala), Schinus fasciculatus (moradillo) y Geoffroea decorticans (chañar), mientras que en el estrato arbustivo dominaron Baccharis salicifolia (chilca) y Lippia turbinata (poleo). A su vez, en esta variante se delimitaron tres categorías:
Bosque secundario cerrado: Dominaron Geoffroea decorticans (chañar) y Vachellia caven (espinillo), además se encontraron Celtis ehrenbergiana (tala), Schinus fasciculatus (moradillo) y Porlieria microphylla (cucharero). En el estrato arbustivo se caracterizó por la presencia de Lycium cestroides Schltdl. (talilla), Lycium ciliatum Schltdl. (piquillín de víbora), Lippia turbinata (poleo), Ephedra triandra (pico de loro), Caesalpinia gilliesii (Wall. ex Hook.) D. Dietr. (lagaña de perro), Cestrum parqui (duraznillo negro), Aloysia gratissima (palo amarillo), Senecio pampeanus Cabrera (sombra de liebre) y enredaderas como Araujia odorata (Hook. \& Arn.) Fontella \& Goyder (tasi), Clematis montevidensis Spreng. (cabello de ángel) y Passiflora caerulea (pasionaria). En el estrato herbáceo abundaron las orquídeas como Sacoila lanceolata (Aubl.) Garay, eudicotiledóneas como Dichondra microcalyx (Hallier f.) Fabris (oreja de ratón), Salpichroa origanifolia (Lam.) Baill. (huevito de gallo), Sphaeralcea crispa Baker f. (malvavisco) y varias especies de poáceas como Botriochloa springfieldii (Gould) Parodi, Chascolytrum subaristatum (Lam.) Desv., Nassella neesiana y Paspalum dilatatum Poir. En los parches con esta variante se pudieron observar renovales de árboles nativos. Esta formación abarcó una superficie de 3285,7 ha $(37,35 \%)$ distribuidas en 99 parches, de los cuales casi un $50 \%$ presentaron superficies menores a 50 ha. Espacialmente, esta formación se encontró distribuida uniformemente a lo largo de toda el área estudiada.

Bosque secundario abierto: Se acentúan los procesos de erosión del suelo por el drenaje del agua de los campos; esta formación se caracterizó por la presencia de árboles bajos aislados de Vachellia caven (espinillo), Lippia turbinata (poleo), y una cobertura vegetal herbácea abundante con cactáceas como Opuntia sulphurea Gillies ex Salm-Dyck, Cereus aethiops Haw. (hachón) y plantas semileñosas como Ephedra triandra (pico de loro). También se observaron Cortaderia selloana (Schult. \& Schult. f.) Asch. \& Graebn. (cortadera), Cyperus rotundus L. (cebollín), además de numerosas especies que actúan como malezas. En las situaciones más degradadas, próximo a los cursos de agua, se registraron 


\section{E. Natale et al. - Caracterización ambiental de la cuenca del río Cuarto}

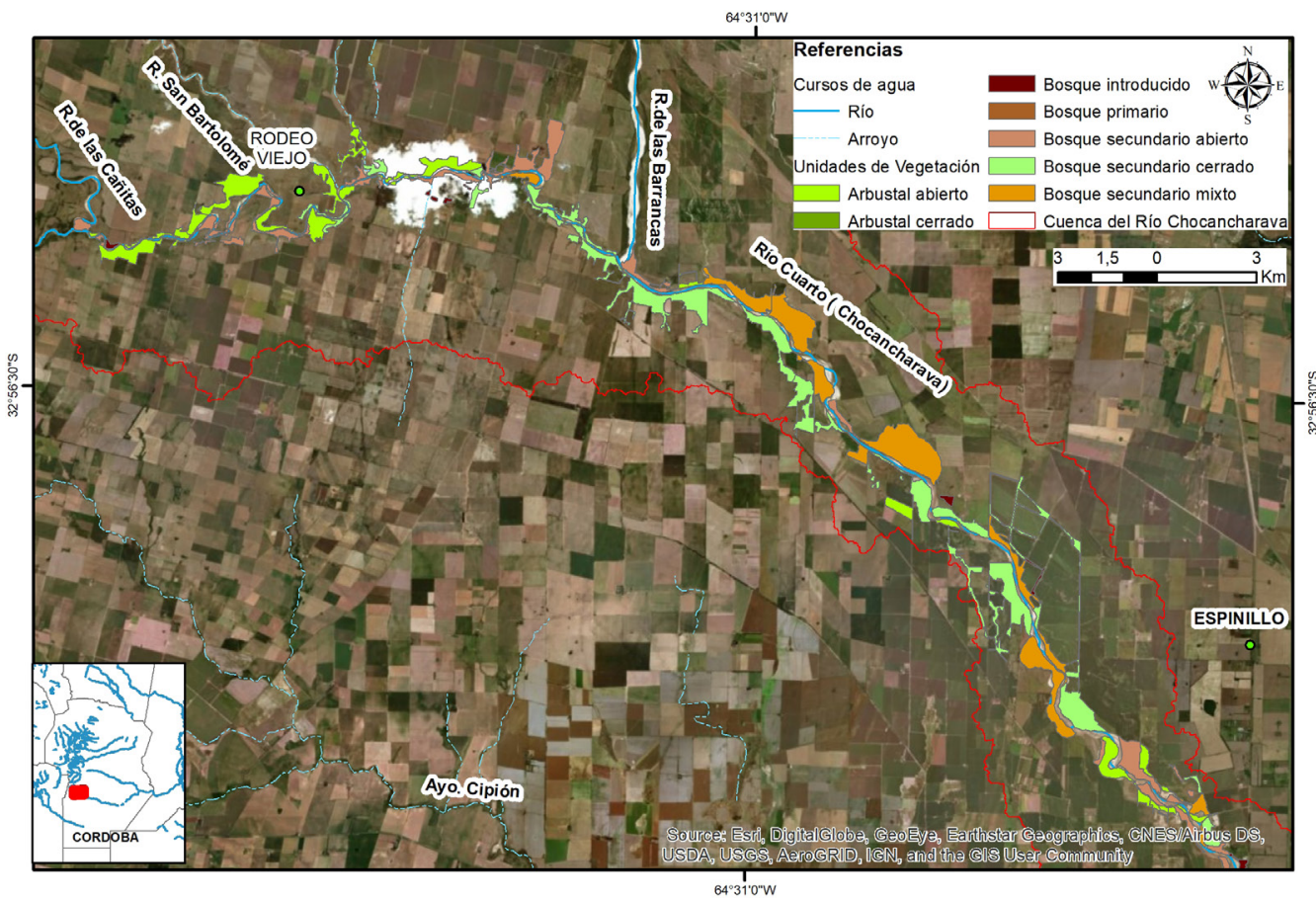

Fig. 2. Unidades de vegetación de la cuenca media del río Cuarto (río Chocancharava). Sector cuenca media-alta.

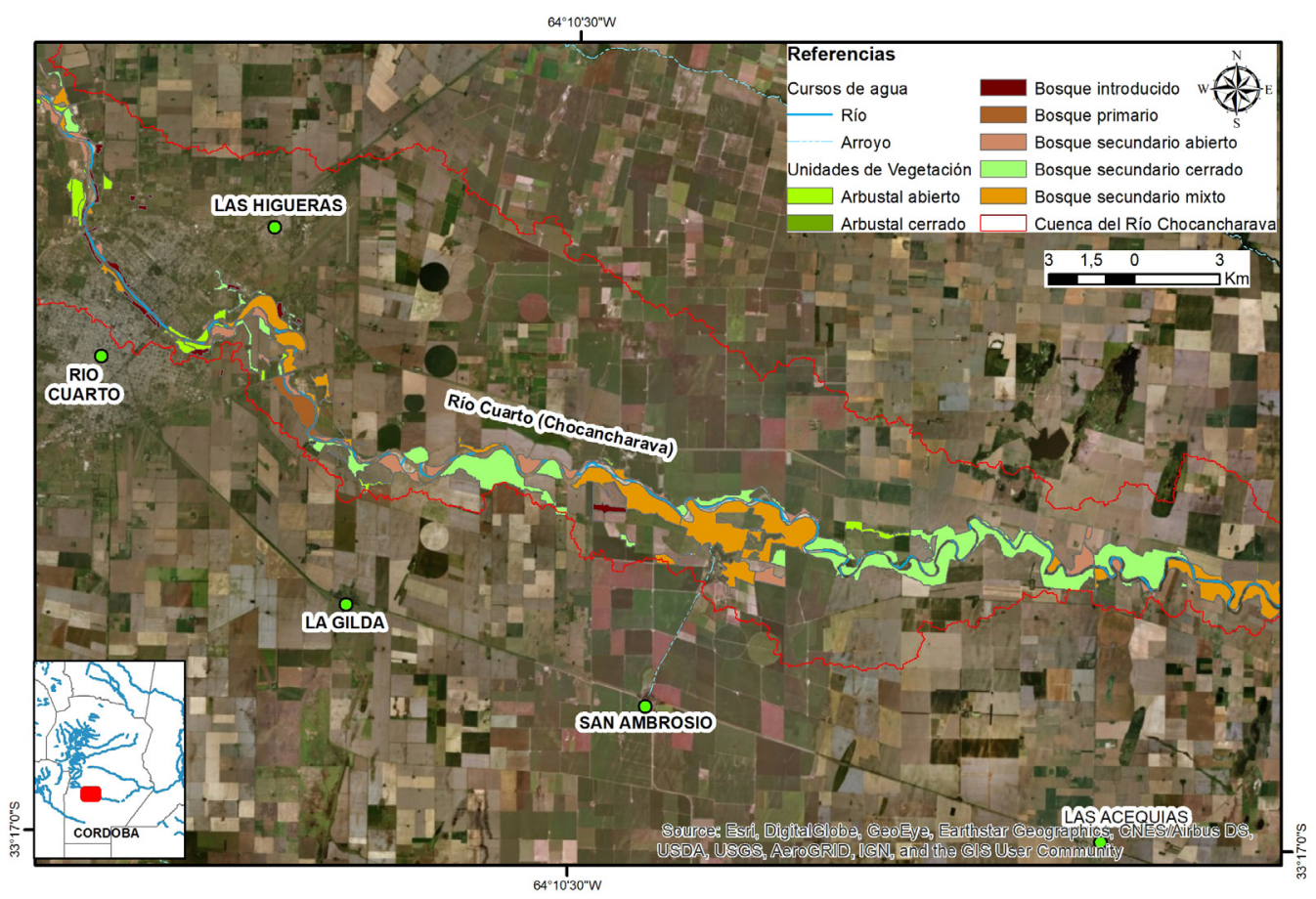

Fig. 3. Unidades de vegetación de la cuenca media del río Cuarto (río Chocancharava). Sector cuenca media-media. 


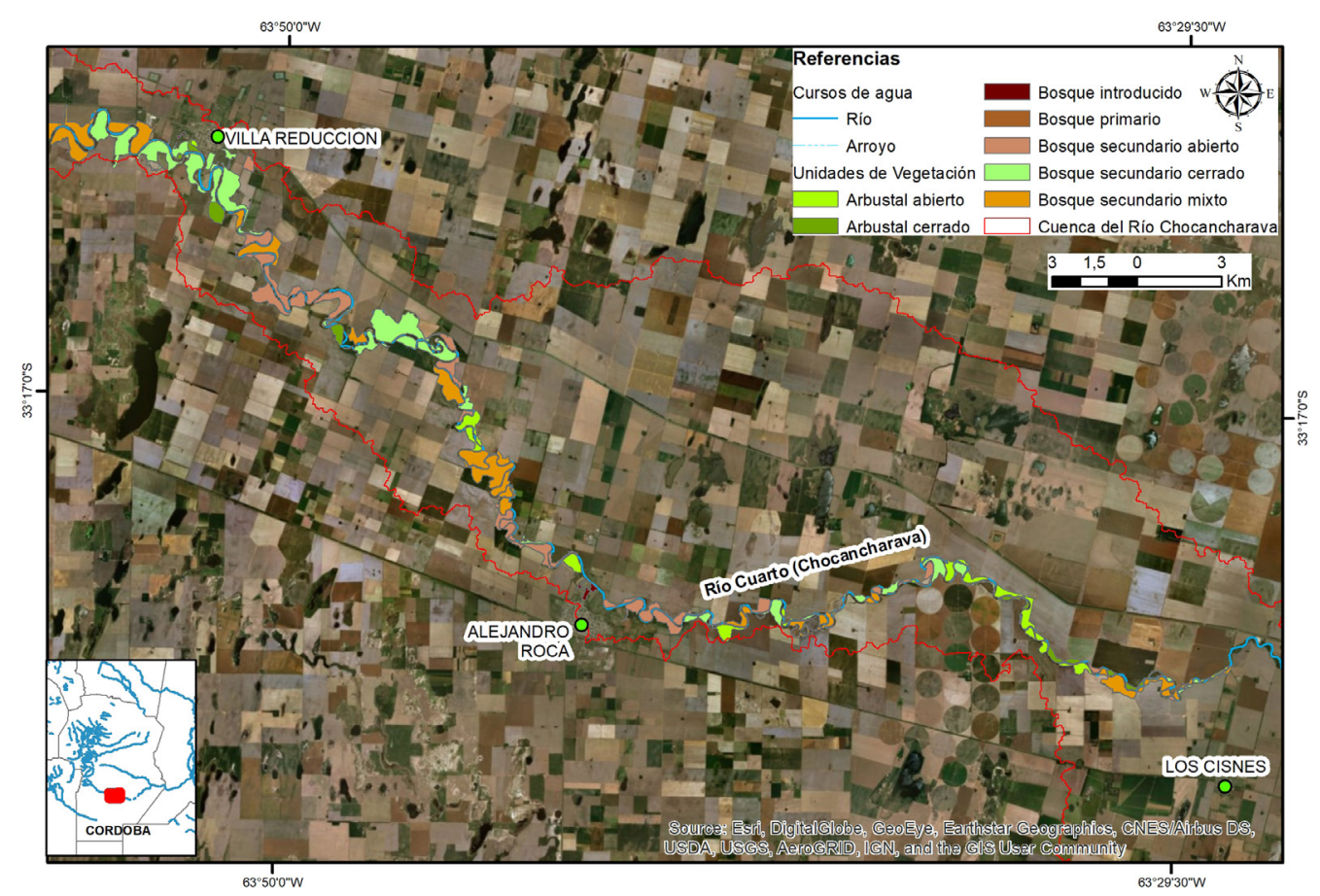

Fig. 4. Unidades de vegetación de la cuenca media del río Cuarto (río Chocancharava). Sector cuenca media-baja.

abundantes matas de especies exóticas como Rubus ulmifolius Schott (zarzamora) y Arundo donax (caña de Castilla). Este tipo de bosques ocupó una superficie de 1388 ha, un $15,79 \%$ del total de la cuenca media del río Chocancharava. Al igual que en la unidad anterior, se presentó fragmentado contabilizando un total de 76 parches distribuidos a lo largo de toda el área de estudio.

Bosque secundario mixto: Formación tipo sabana con predominio de cobertura vegetal herbácea del suelo y solo algunos ejemplares leñosos aislados de Vachellia caven (espinillo), Geoffroea decorticans (chañar), Porlieria microphylla (cucharero) y Celtis ehrenbergiana (tala). En estos ambientes se entremezclan abundantes arboledas de especies exóticas compuestas por Ligustrum lucidum (siempre verde), Ulmus pumila (olmo), Melia azedarach (paraíso), Gleditsia triacanthos (acacia negra) y Robinia pseudoacacia L. (acacia blanca). Los bosques mixtos presentaron una superficie de 2655,8 ha $(30,18 \%)$ distribuidas en 56 parches comenzando a la altura de río Seco hasta la localidad de Los Cisnes.

Bosque Introducido: donde los árboles existentes pertenecen a especies exóticas como Ligustrum lucidum (siempre verde), Melia azedarach (paraíso), Gleditsia triacanthos (acacia negra), Ulmus pumila (olmo), Broussonetia papyrifera (morera de papel) y Salix fragilis (sauce frágil). Esta unidad solo abarcó unas 201,15 ha, un $2,28 \%$ de la superficie total estudiada distribuida en 39 parches de pequeño tamaño, menor a 23 ha. A diferencia de las unidades anteriores, estos bosques se concentraron en los alrededores de las zonas urbanas, principalmente sobre la ciudad de río Cuarto.

Arbustal: área en donde las plantas dominantes fueron arbustos leñosos acompañados de vegetación herbácea de diferentes portes con neto dominio de gramíneas, sin árboles. En este tipo se delimitaron dos variantes:

Arbustal abierto: ambientes con predominio de pastos y con escasos arbustos aislados de 


\section{E. Natale et al. - Caracterización ambiental de la cuenca del río Cuarto}

Baccharis salicifolia (chilca). Se encontró dispuesto en unos 60 parches que ocuparon una superficie de 1058,7 ha, un $12,03 \%$ del total de la cuenca media. Al igual que el Bosque introducido tuvieron una configuración espacial asociados a las zonas urbanas.

Arbustal cerrado: predominio de vegetación arbustiva leñosa cubriendo con individuos de las especies Lippia turbinata (poleo), Cortaderia selloana (cortadera), Baccharis salicifolia (chilca) y Aloysia gratissima (palo amarillo). Solo se presentó en la cuenca media baja, a partir de la localidad de Reducción, con tan solo 8 parches, muy aislados entre sí, que sumaron unas 108 ha $(1,22 \%)$.

\section{Estado de conservación de la cuenca media del río Cuarto}

Para ajustar el desempeño de Índice Integral de Evaluación en el área de estudio se calibró el componente geológico (Valor de singularidad geológica + valor de protección - ver anexo I) definiendo nuevas variables que se aplicaran al área de estudio. En base a esto, se tomó como "valor de singularidad" a la capacidad del río de generar humedales, es decir que mediante el mecanismo de desborde se formen llanuras aluviales inundadas y funcionen como niveles de almacenamiento transitorio de agua. Mientras que, para determinar el "valor de protección", se consideraron tres variables: 1) la relación agua superficial - agua subterránea que considera la conexión existente entre el curso de agua del río y el acuífero freático que se encuentra en el subsuelo; 2) la erodabilidad de márgenes, definida como la susceptibilidad de la unidad ambiental a ser erosionada debido a la dinámica fluvial; y 3) la pendiente. Para todas estas variables se definieron los umbrales críticos siguiendo la metodología presentada por Natale et al. (2015), para poder ser incorporadas al cálculo del IIEA (Tabla 1).

Una vez ajustadas las variables geológicas al área de estudio, se procedió a correr el Índice Integral de Evaluación Ambiental para cada una de las unidades ambientales definidas. Así el IIEA arrojó que el 56,5\% (192) de las unidades ambientales se encontraron en mal estado de conservación, el 31,2\% (106) presentaron un estado regular, el 11,8\% (40) alcanzó un buen estado y tan sólo un 0,6\% (2) llegó a un muy buen estado de conservación (Fig. 5-7). Por otro lado, los resultados de la variable que midió la funcionalidad ecológica arrojaron que el 65\% (221) de las unidades ambientales fueron consideradas corredores biológicos. La distancia promedio al parche más cercano fue de $3,92 \mathrm{~m}$, aunque se reportaron algunos casos con distancia mayores a los $500 \mathrm{~m}$, estas mayores distancias entre parches se ubicaron espacialmente asociadas a los conos urbanos.

En la Tabla 2 se muestra el estado de conservación por unidad de vegetación donde se destaca que las dos únicas unidades ambientales de bosque primario presentaron estados regular y bueno. Por otro lado, el bosque secundario mixto fue el que incluyó las dos unidades ambientales en muy buen estado de conservación (Fig. 6).

Para la ejecución del análisis de componentes principales categóricos se excluyeron las unidades ambientales (UA) en muy buen estado de conservación ya que, por encontrarse en tan baja proporción, son consideradas como "outlayer" por el programa. Dicho análisis generó como resultado que la variable de

Tabla 1. Valores umbrales de las variables geológicas definidas para las variables de susceptibilidad y singularidad geológica.

\begin{tabular}{|c|c|c|c|}
\hline Susceptibilidad & Alta & Media & Baja \\
\hline Erodabilidad de márgenes & Muy erodable & Parcialmente resistentes & Resistente \\
\hline Pendiente & $>30 \%$ & $30-10 \%$ & $<10 \%$ \\
\hline $\begin{array}{l}\text { Relación agua superficial- } \\
\text { agua subterránea }\end{array}$ & $\begin{array}{l}\text { Freática influente o efluente } \\
\text { con cursos superficiales }\end{array}$ & & Indiferente \\
\hline \multicolumn{4}{|l|}{ Singularidad } \\
\hline Potencialidad de ser Humedal & $\begin{array}{l}\text { Área que se anegan } \\
\text { periódicamente }\end{array}$ & Área de desborde & $\begin{array}{c}\text { Baja o nula probabilidad } \\
\text { de desborde }\end{array}$ \\
\hline
\end{tabular}


Bol. Soc. Argent. Bot. 54 (1) 2019

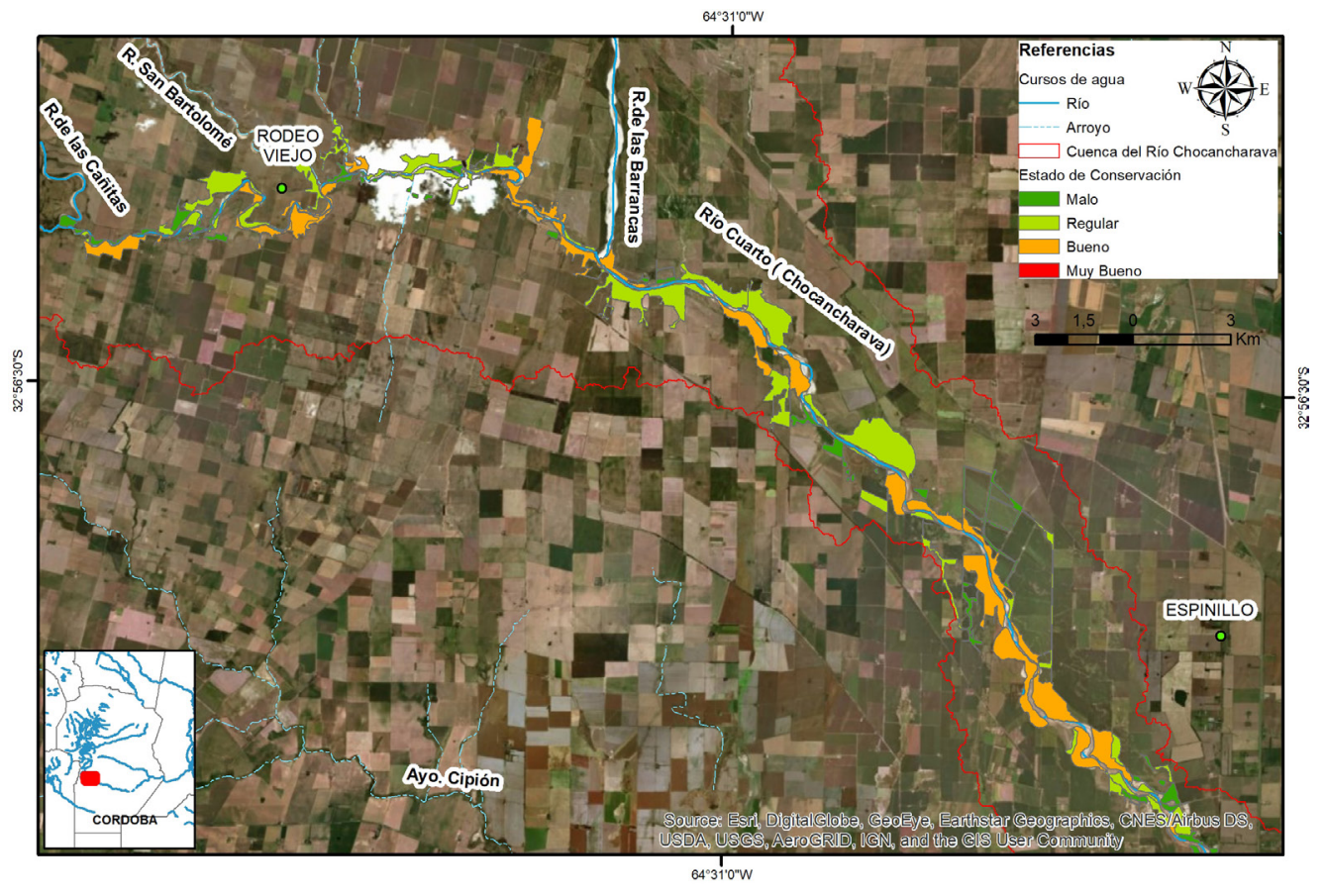

Fig. 5. Estado de Conservación de las unidades ambientales asociadas a la cuenca media del río Cuarto (río Chocancharava). Sector cuenca media-alta.

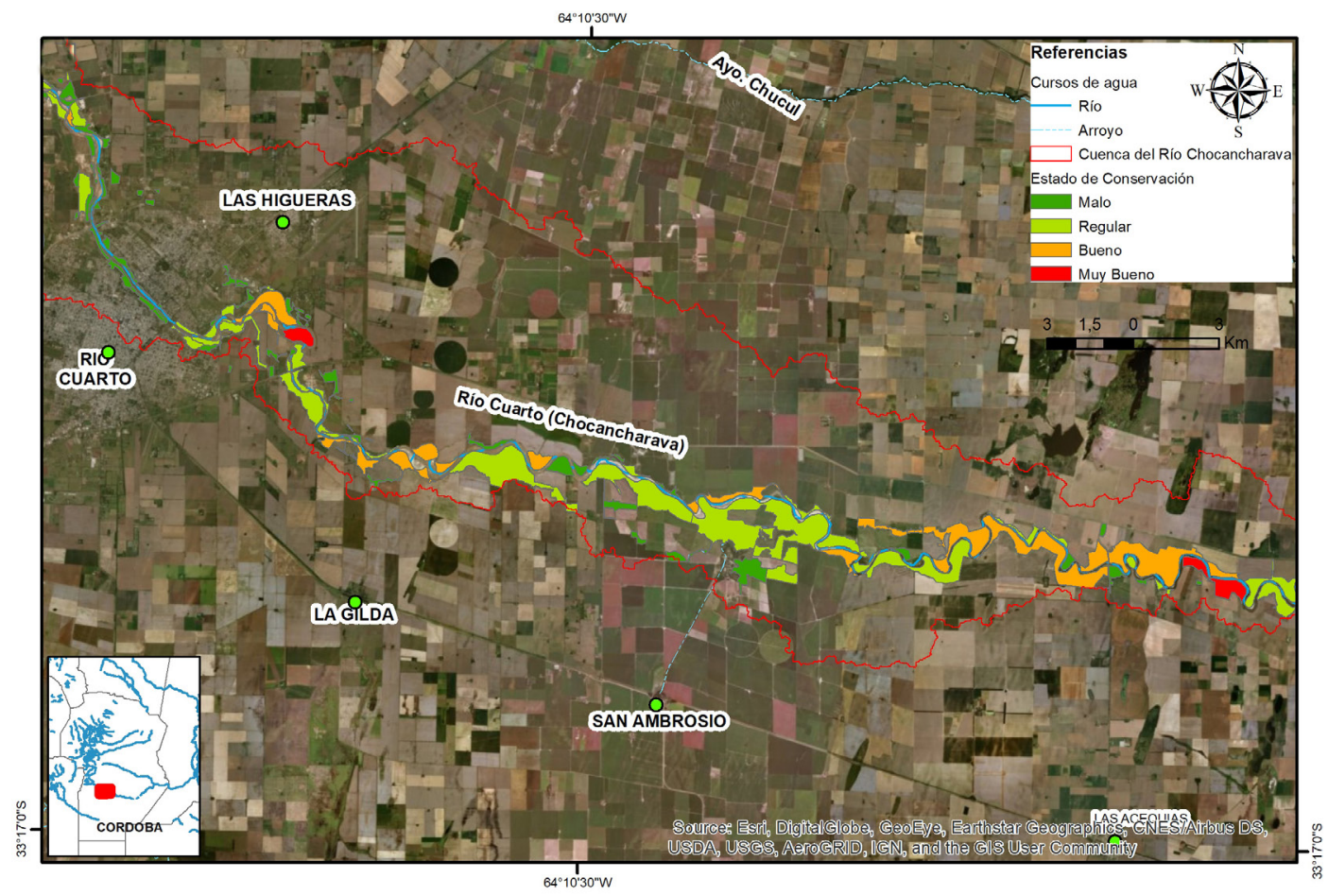

Fig. 6. Estado de Conservación de las unidades ambientales asociadas a la cuenca media del río Cuarto (río Chocancharava). Sector cuenca media-media. 


\section{E. Natale et al. - Caracterización ambiental de la cuenca del río Cuarto}

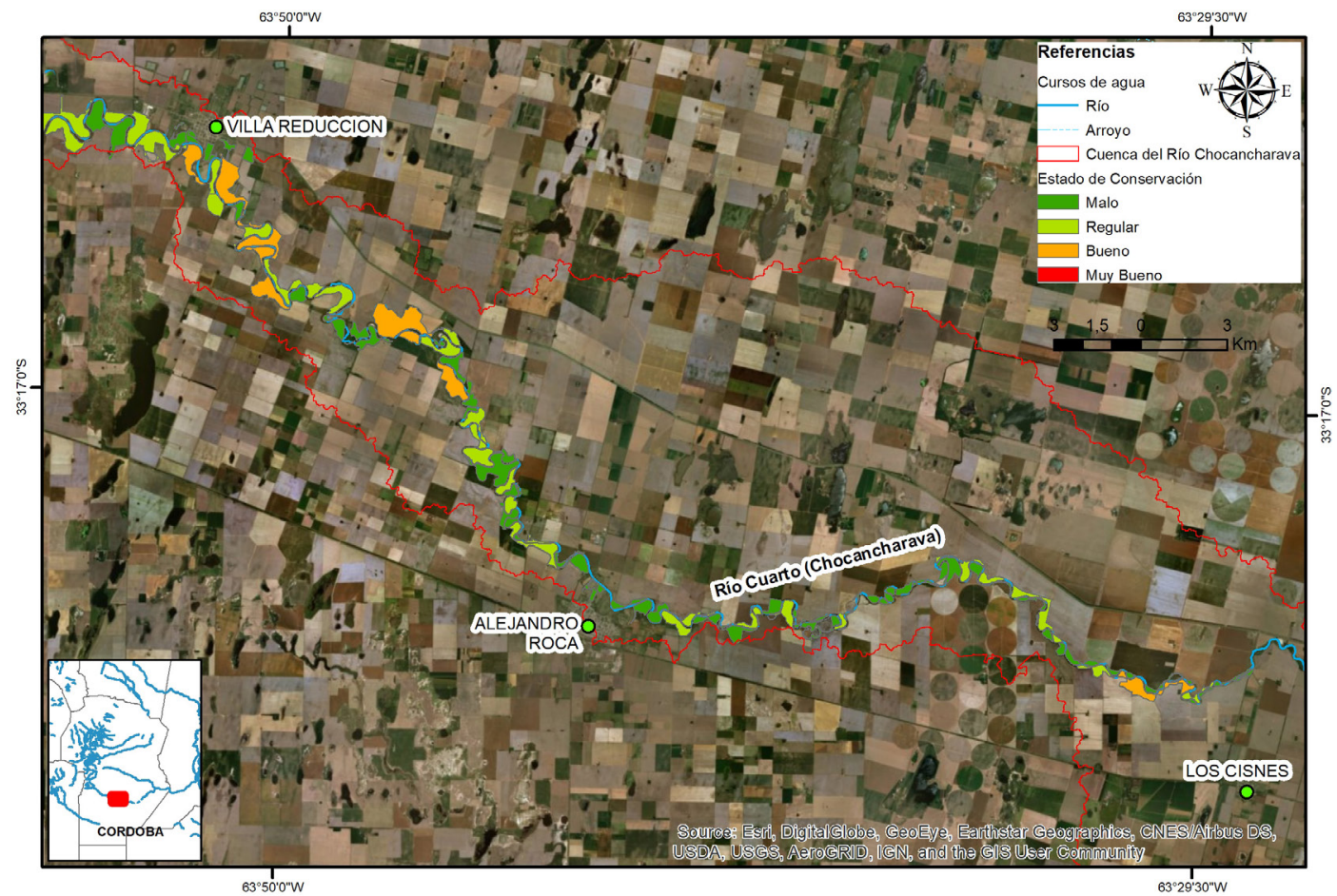

Fig. 7. Estado de Conservación de las unidades ambientales asociadas a la cuenca media del río Cuarto (río Chocancharava). Sector cuenca media-baja.

impacto antrópico que separa a las UA en mal estado de conservación del resto fue la superficie invadida, mostrando valores medios de invasión (16 al 30\%) como la situación más frecuente. El resto de los impactos, con sus diferentes grados, se distribuyeron de manera variable entre las tres categorías (Fig. 8).

En cuanto a la integridad ecológica, la variable tamaño del parche separó a las UA en mal estado de conservación del resto de las categorías, indicando que el mayor porcentaje de UA en mal estado se da por presentar superficies menores a 10 ha. El resto de las variables de integridad ecológica no pudieron explicar los diferentes estados de conservación mostrando una gran uniformidad en los valores a lo largo de todo el trayecto estudiado. La mayoría de los parches presentaron formas alargadas y contactos

Tabla 2. Cantidad de unidades de vegetación, de la cuenca media del río Cuarto, en diferentes categorías de conservación.

\begin{tabular}{|cccccc|}
\hline Unidad de Vegetación & $\begin{array}{c}\text { Malo } \\
\mathrm{N}^{\circ}(\%)\end{array}$ & $\begin{array}{c}\text { Regular } \\
\mathrm{N}^{\circ}(\%)\end{array}$ & $\begin{array}{c}\text { Bueno } \\
\mathrm{N}^{\circ}(\%)\end{array}$ & $\begin{array}{c}\text { Muy Bueno } \\
\mathrm{N}^{\circ}(\%)\end{array}$ & $\begin{array}{c}\text { Total } \\
\mathrm{N}^{\circ}\end{array}$ \\
\hline Arbustal abierto & $31(52)$ & $24(40)$ & $5(8)$ & 0 & 60 \\
Arbustal cerrado & $6(75)$ & $2(25)$ & 0 & 0 & 8 \\
Bosque primario & 0 & $1(50)$ & $1(50)$ & 0 & 2 \\
\hline Bosque secundario abierto & $40(53)$ & $26(34)$ & $10(13)$ & 0 & 76 \\
Bosque secundario cerrado & $50(51)$ & $27(27)$ & $22(22)$ & 0 & 99 \\
Bosque secundario mixto & $23(41)$ & $20(36)$ & $11(20)$ & $2(4)$ & 56 \\
Bosque introducido & $39(100)$ & 0 & 0 & 0 & 39 \\
\hline
\end{tabular}


Bol. Soc. Argent. Bot. 54 (1) 2019

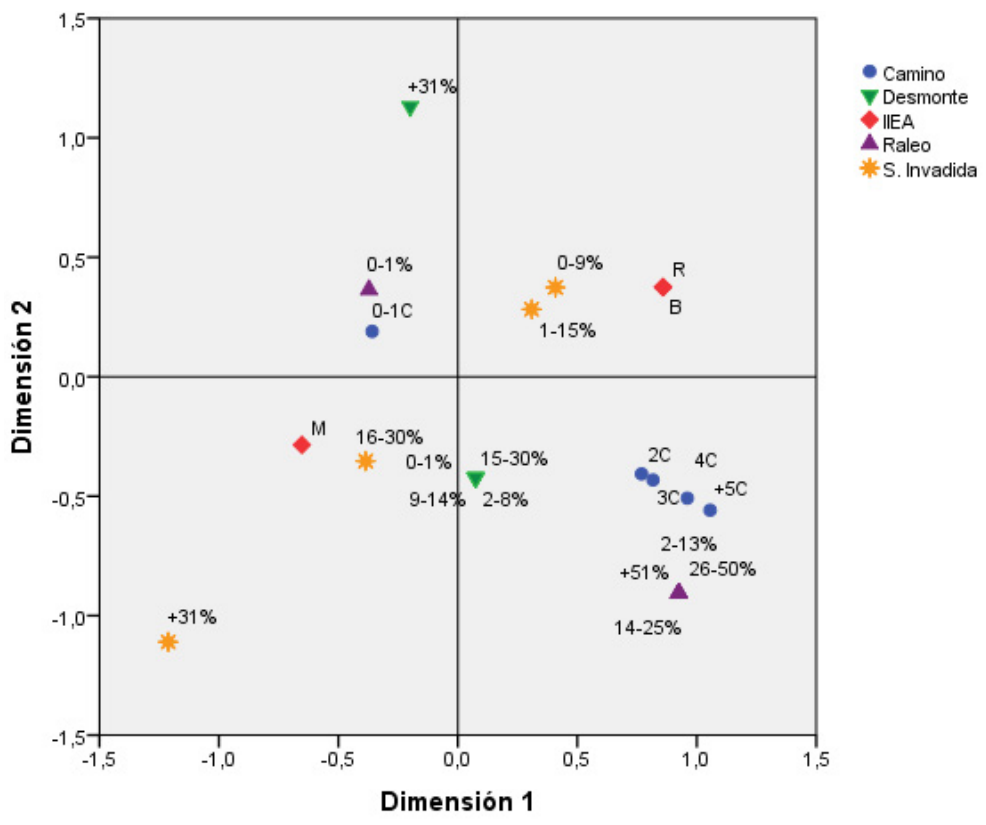

Fig. 8. CCA categóricos con las variables de contexto utilizadas en el Índice Integral de Evaluación Ambiental para las UA pertenecientes al río Chocancharava.

estructurales naturales, por encontrarse dentro de la faja fluvial del curso de agua por un lado, y contactos antrópicos generados por el avance de la frontera agrícola hasta donde lo permite la estabilidad del terreno (Fig. 9). En el mismo sentido las variables geológicas mostraron que las UA con categorías

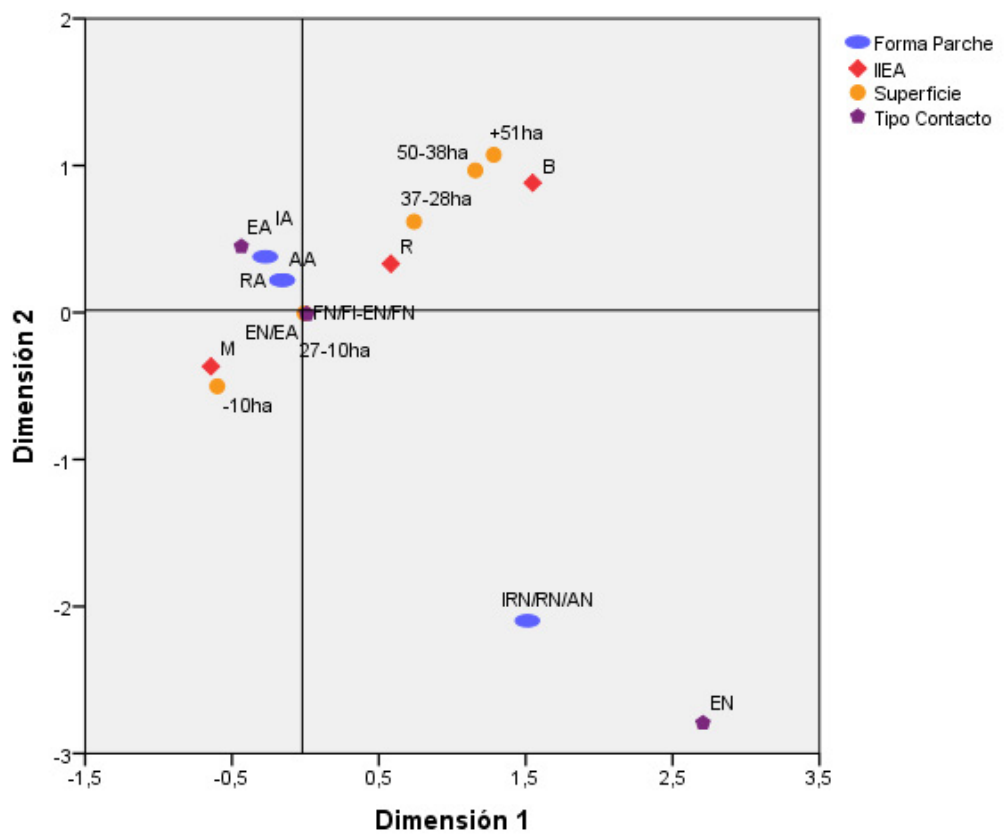

Fig. 9. CCA categóricos con las variables de integridad ecológica utilizadas en el Índice Integral de Evaluación Ambiental para las UA pertenecientes al río Chocancharava. 


\section{E. Natale et al. - Caracterización ambiental de la cuenca del río Cuarto}

de regular a bueno se relacionaron con altos valores de erodabilidad. Respecto a la variable de singularidad, la potencialidad de funcionar como humedal, las UA regulares y buenas presentaron valores de medio a alto, siendo las UA de estado de conservación regular las que presentaron valores más altos en cuanto a la potencialidad de funcionar como un humedal. A esto se suma que tanto la relación de influencia como de efluencia entre el agua superficial y el agua subterránea también se mostró más asociada con las UA regulares y buenas. Estas mismas categorías también estarían funcionando como corredores biológicos (Fig. 10). Es importante aclarar que de los tres componentes del Índice Integral de Evaluación Ambiental el que mayor poder de explicación de la varianza tuvo fue el componente de contexto (impacto antrópico) explicando un $58,74 \%$.

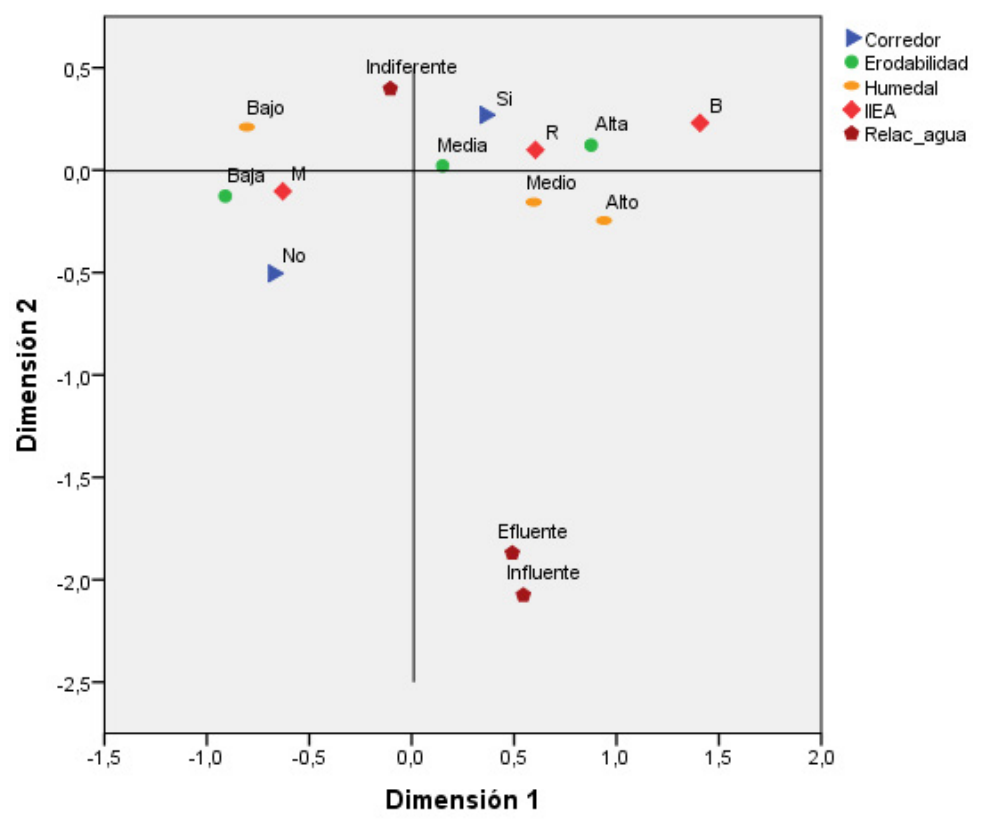

Fig. 10. CCA categóricos con las variables geológicas utilizadas en el Índice Integral de Evaluación Ambiental para las UA pertenecientes al río Chocancharava.

\section{Discusión y Conclusión}

Debido al avance de la frontera agrícola en toda la cuenca media del río Cuarto, los únicos relictos de vegetación que quedan son los que se encuentran asociados a las márgenes del río Chocancharava; por lo que se puede decir que tan sólo el $8 \%$ de la superficie de la cuenca media se encuentra cubierta por vegetación nativa, entre bosques y arbustales en distintos grados de conservación, y más del $50 \%$ de los parches presentan altos grados de degradación antrópica. Tan solo 139 ha (2 UA), que representan menos del $0,6 \%$ de la superficie estudiada, pudieron ser consideradas de alto valor de conservación (Figs. 6; 7). Cabe destacar que ambas UA no fueron las que presentaron mayores tamaños, sino las que exhibieron valores casi nulos de superficie invadida y, en general, valores bajos respecto a los impactos antrópicos como presencia de caminos y asentamientos, superficies raleadas y/o desmontadas. Sumado a esto, se encontraron alejadas de las zonas urbanas y rodeadas de UA en estado de conservación de bueno a regular, que podrían estar actuando como zonas de amortiguamiento frente a los impactos antrópicos. Estos resultados apoyan lo expresado por Frank et al. (2012) acerca de la necesidad de incorporar, a este tipo de estudios, variables que contemplen y valoren, no solo la extensión del parche, sino también su configuración espacial y el contexto que los rodea. 
Los mayores impactos sobre los ambientes estudiados fueron el avance de las especies exóticas invasoras y la drástica disminución de la superficie por avance del desarrollo urbano y agroproductivo; esto dejó un gran porcentaje de unidades ambientales con superficies menores a 10 ha y con procesos de invasión que han reemplazado casi totalmente a la vegetación nativa. Por otro lado, la existencia de parches dentro de categorías de conservación regulares a buenas, con tamaños mayores a 51 ha y altas probabilidades de funcionar como humedales temporarios y con graves procesos de erosión, se debió a que presentaron una considerable superficie (hasta un 15\%) con procesos de invasión. Estos resultados sostienen lo expuesto por el Programa de las Naciones Unidas para el Ambiente (UNEP), quienes consideran que las invasiones biológicas representan el mayor factor de presión para el 15\% de las especies de plantas en riesgo de extinción, y se ha convertido en la segunda amenaza mundial para la biodiversidad en sistemas aislados tanto geográfica como evolutivamente (Kairo et al., 2003). En este sentido, investigaciones realizadas en Chile (Romero et al., 2014) han mostrado que las microcuencas con porcentajes mayores de cobertura con plantaciones exóticas, producen un menor caudal hídrico estival que las cuencas con una cubierta mayor de especies nativas, afectando a las actividades productivas.

La necesidad de evaluar la calidad de los cursos de agua ha llevado al desarrollo de una cantidad considerable de índices para la valoración de la vegetación de ribera. Así, se pueden nombrar al índice "QBR" desarrollado por Munné et al., (1998), el índice RQI (Riparian Quality Index) (Gonzales del Tánago et al., 2006), el IFV (Índice de Vegetación Fluvial) (Gutierrez et al., 2001) y el RFV (Evaluación del estado del bosque de ribera) desarrollado por Magdaleno et al. (2010), entre otros. Todos ellos se basan en la recopilación de diferentes atributos de los componentes del área de ribera; algunos haciendo más énfasis en la estructura de la vegetación, otros en la composición, en la continuidad del bosque a lo largo del curso de agua y en la capacidad de regeneración del sistema. En una comparación entre el índice "QBR" y el IIEA, utilizado en este estudio, consideramos que éste último presenta algunas posibles ventajas. En primer lugar, la asignación de los valores para cada una de las variables en el índice QBR depende de una evaluación visual, mientras que el IIEA se basa en índices de aptitud (SI) de cada variable, con umbrales asignados por fundamentos teóricos de la biología de la conservación y la ecología del paisaje. Sumado a esto, el IIEA incluye variables de impacto antrópico que el QBR no contempla. Si se compara el resto de los índices con el aplicado en este estudio, de manera general, se puede decir que la mayoría presenta un valor final que surge de la sumatoria de las variables evaluadas, mientras que el IIEA presenta un algoritmo que intenta reflejar las interacciones ecológicas entre los componentes del sistema y ponderar las variables, de acuerdo a la influencia que pueden llegar a ejercer sobre el estado del mismo. Finalmente, el IIEA, permite evaluar grandes áreas en poco tiempo y expresar los resultados en un entorno cartográfico, fundamental en el ámbito de la planificación territorial.

De esta manera, se concuerda con lo expuesto por Natale et al. (2015) respecto a que el índice, como herramienta, permite abarcar grandes extensiones, ya que en esta oportunidad se logró evaluar una superficie de 302,832 ha en un periodo relativamente corto de tiempo y a un bajo costo. Sumado a esto, la obtención de un valor cuantitativo de conservación, a pesar de que en la representación cartográfica se haya expresado con valores categóricos, brindará, a los agentes de planificación urbana y ordenamiento territorial, un orden de prioridad a la hora de ejecutar estrategias de intervención del territorio. Por un lado, se podrán priorizar las acciones de restauración comenzando con las unidades ambientales que posean mayor posibilidad de mantener procesos ecosistémicos; $\mathrm{y}$, por otro lado, frente al inminente avance de los procesos de desarrollo, se podrá definir áreas denominadas "de sacrificio", considerando las unidades ambientales con los valores más bajo de índice y que por ende menos factibles (económica y logísticamente) de ser recuperadas.

Según Gomez Orea \& Gomez Villarino (2013), en cualquier propuesta de ordenamiento territorial se deben incluir las tres dimensiones del desarrollo sustentable: albergar a más población, reducir el impacto económico y la pérdida de servicios ecosistémicos y, finalmente, minimizar el esfuerzo político institucional necesario para realizar la propuesta. De acuerdo a los resultados obtenidos en este trabajo, y coincidiendo con lo postulado por los mencionados autores, se resalta la necesidad de 


\section{E. Natale et al. - Caracterización ambiental de la cuenca del río Cuarto}

incorporar en los planes de ordenamiento territorial el componente ambiental, que lleve a un desarrollo urbano que minimice los costos económicos, ambientales y sociales, permitiendo mejorar la calidad de vida de los habitantes.

En cuanto a la actuación del índice en diferentes situaciones ambientales, podemos decir que los resultados obtenidos aquí (56\% de UA en mal estado de conservación) fueron sustancialmente diferente a lo obtenido por Natale et al. (2015), a partir de la aplicación del mismo índice en los sistemas naturales de la ciudad de Merlo (provincia de San Luis), donde el 60\% de las UA se encontraron en muy buen estado de conservación. Esta gran diferencia es atribuible a que los sectores estudiados pertenecen a cuenca media y cuenca alta respectivamente $\mathrm{y}$, por lo tanto, sometidos a diferentes intensidades de uso del suelo, situación que el IIEA estaría reflejando. Sumado a esto, el presente estudió arrojó un alto porcentaje de UA en mal estado de conservación, que coincidieron con las formaciones de arbustales consideradas etapas tempranas de una sucesión secundaria, producto de procesos de recuperación natural del sistema. Situación que se presentó de manera opuesta en el trabajo realizado por Natale et al. (2014) en la zona de cuenca alta del río Cuarto donde el $81 \%$ de las formaciones de arbustal, uno de los estadíos maduros de sucesión en estos ambientes, se encontró en muy buen estado de conservación. Ambas comparaciones proporcionan evidencias sobre una buena performance del IIEA como herramienta de evaluación a nivel de paisaje, que permitiría la comparación entre diferentes situaciones ambientales.

Finalmente, de acuerdo a la meta siete (7) del Plan estratégico de conservación de la diversidad biológica 2011-2020 y las metas AICHI, que expresan que las zonas destinadas a agricultura, acuicultura y silvicultura se gestionarán de manera sostenible, garantizándose la conservación de la diversidad biológica, de los resultados de este trabajo se pueden puntualizar algunas consideraciones $\mathrm{y}$ recomendaciones de manejo a tener en cuenta al momento de la planificación del territorio de las ciudades asociadas al río Chocancharava, de manera de propender al cumplimiento de dichas metas.

Debido al alto porcentaje de parches en mal estado de conservación, se deben aplicar estrategias curativas tendientes a la recuperación de los factores ambientales y de las áreas degradadas; mientras que, en los parches con bueno a muy buen estado de conservación, deberían aplicarse estrategias que permitan fortalecer la homeostasis y resiliencia de sus componentes. A partir del análisis de las variables utilizadas, se pudo agrupar la totalidad de los parches estudiados en categorías de intervención, teniendo en cuenta su importancia relativa en el sistema cuenca, el estado de conservación que arrojó el IIAE y el costo logístico de llevar a cabo las acciones de recuperación. Así se definieron cuatro grupos.

Grupo I (UA en mala condición): corresponden a tamaños pequeños con graves procesos de invasión y altamente degradados; a esto se suma que se encuentran en zonas de bajo valor de protección y singularidad, inmersas en áreas urbanas y asociadas a zonas de usos recreativos. Las estrategias se deben centrar en la utilización de las especies arbóreas nativas para la forestación de las zonas de uso recreativo como así también la valoración de las mismas dentro el arbolado público de las ciudades. Esto permitirá disminuir la presión de propágulos y posterior dispersión de especies exóticas invasoras hacia unidades ambientales en mejores estados de conservación. A esto se tienen que sumar acciones específicas de control de Especies Exóticas Invasoras. En este sentido, a través de la participación de los investigadores, autores de este trabajo, en el Consejo Consultivo Municipal de Protección del Arbolado Público de la ciudad de río Cuarto, se logró aprobar la Ordenanza Municipal 558/17 “especies recomendadas y prohibidas para el arbolado público urbano para la ciudad de río Cuarto" donde se recomiendan especies nativas para la forestación y se prohíbe la utilización de especies exóticas invasoras como el olmo y el ligustro, y otras con antecedentes de invasión en la provincia.

Grupo II (UA en condición regular a mala; estas últimas de superficies mayores a 20 ha concentradas en la zona sur de la cuenca): En general presentan grandes superficies por lo que las acciones deben centrarse en controlar la actividad antrópica principal y/o mitigar amenazas incipientes. La mayoría de las actividades antrópicas se vinculan a actividades no permitidas como el depósito de residuos sólidos urbanos, de construcción o poda, la extracción indiscriminada de áridos, la proliferación 
de pistas de motocross, entre otras. Estas actividades deben ser reguladas y/o erradicadas con un efectivo control y fiscalización de la zona por parte de los gobiernos tanto provinciales como municipales.

Grupo III (UA en condiciones regulares, con superficies mayores a 51 ha, a buenas): Estos parches se presentan como áreas de más de 60 ha con formas alargadas, en su mayoría, configurándose en el paisaje como verdaderos corredores biológicos. Sumado a esto están emplazados en zonas con alto valor de protección y singularidad. Por tal motivo las estrategias de manejo deben centrarse en medidas de conservación eficaces fuera de áreas protegidas, tales como reservas privadas, regulación de usos, entre otras. En este sentido la Ciudad de río Cuarto, a través de la ordenanza 183/12, declaró al área coincidente con la unidad geomorfológica faja fluvial del río Cuarto como zona de Preservación y lo denominó Corredor Ambiental Chocancharava. Es importante recordar que en esta categoría entran las dos UA pertenecientes a bosque primario por lo que, debido a su alta riqueza taxonómica y funcional debería considerarse una figura legal de conservación que permita resguardar los componentes originales del espinal, principalmente el bosque de quebrachos más austral de la Argentina.

Teniendo en cuenta estos resultados, se puede resaltar un aspecto metodológico respecto al índice utilizado, en cuanto a que el mismo no tuvo la sensibilidad suficiente para discriminar aquellos parches con singularidad biológica, particularmente en este caso debido a sus componentes botánicos (presencia de quebrachos). Por lo que sería interesante explorar la posibilidad de incorporar este tipo de variables con la finalidad de ponderar elementos particulares de la biodiversidad

Grupo IV (UA en muy buen estado de conservación): Debido a que solo dos de las 340 unidades relevadas presentan esta condición, la estrategia más adecuada debería ser la conservación estricta a través de la creación de una figura legal de protección. Aquí es importante destacar que los resultados de este estudio fueron plasmados en una propuesta de creación de área protegida que fue avalada por la Mesa Interinstitucional del corredor Chocancharava (creada en diciembre de 2017) y el Concejo Deliberante de la ciudad de río Cuarto, y presentada ante la legislatura de la provincia de Córdoba. Fue así que, en marzo de 2018, se creó por ley provincial 10.525 la "Reserva Provincial de Uso Múltiple Corredor Chocancharava". La misma incluye la Unidad ambiental en muy buen estado de conservación que se encuentra al sur de la ciudad de río Cuarto, las tres UA en buen estado de conservación que se encuentran más al norte y las dos UA en estado regular que se encuentran hacia el sur, siendo el limite el puente Antártida Argentina, emplazado sobre la Ruta Nacional $\mathrm{N}^{\circ}$ 8. La creación de esta reserva, de más de 230 ha, permitió aumentar la superficie bajo protección del distrito biogeográfico el espinal, una de las regiones más amenazadas por la expansión agrícola (Brown et al., 2005; Oggero et al., 2014) y con menos del $5 \%$ de superficie protegida bajo alguna figura legal (Bertonatti \& Corcuera, 2000).

La elaboración e implementación de estas recomendaciones tienden a cumplir con la Meta 14 de AICHI y la meta 21 de la Estrategia Nacional Sobre Biodiversidad, en donde, en líneas generales, se plantea que para el 2020 se deberán haber restaurado y salvaguardado los ecosistemas que proporcionan servicios esenciales, incluidos servicios relacionados con el agua, y que contribuyen a la salud, los medios de vida y el bienestar (MAyDS, 2016).

\section{Contribución DE LOS AUtORES}

EN:Confeccióndecartografía debase, digitalización de las Unidades Ambientales, clasificación visual mapa de vegetación, muestreos a campo, ejecución del Índice Integral de Evaluación Ambiental, análisis de componentes principales, evaluación de resultados para diseño de recomendaciones de manejo. MA, AO: Censos de vegetación, definición de unidades de vegetación, identificación de especies. GV: Muestreos a campo, definición de variables geológicas, valoración del componente geológico de las unidades ambientales, evaluación de resultados para diseño de recomendaciones de manejo. MDLR: muestreos a campo, valoración de las unidades ambientales respecto a las variables del IIEA. HR: Revisión general del manuscrito, directora del proyecto.

\section{Agradecimientos}

Evangelina Natale recibe financiamiento de CONICET (Consejo Nacional de Investigaciones 


\section{E. Natale et al. - Caracterización ambiental de la cuenca del río Cuarto}

Científicas y Técnicas, Argentina). Este trabajo fue financiado por la SecyT - UNRC. Agradecemos a los miembros de la Mesa del Corredor Chocancharava por su compromiso e involucramiento en la implementación de los resultados de este estudio en estrategias concreta de conservación. Finalmente, un especial agradecimiento a la Geóloga Gabriela Villalba quien dedicó su vida a los estudios aplicados a la conservación de los recursos naturales de nuestro país, a cuya memoria se dedica este trabajo.

\section{Biblografía}

ARANA, M. D. \& C. A. BIANCO. 2011. Helechos y licofitas del centro de la Argentina. Editorial UNRC.

ARANA, M. D., G. MARTINEZ, A. J. OGGERO, E. NATALE \& J. J. MORRONE. 2017. Map and shapefile of the biogeographic provinces of Argentina. Zootaxa 4341: 420-422.

https://doi.org/10.11646/zootaxa.4341.3.6

BENTON, T. G., J. A. VICKERY \& J. D. WILSON. 2003. Farmland biodiversity: is habitat heterogeneity the key?. Trends Ecol. Evol.18: 182-188. https://doi.org/10.1016/S0169-5347(03)00011-9

BERTONATTI, C. \& J. CORCUERA. 2000. Situación ambiental argentina 2000. Fundación Vida Silvestre Argentina. Buenos Aires. Argentina.

BILENCA D., M. CODESIDO \& C. GONZÁLEZ FISCHER. 2008. Cambios en la fauna Pampeana. Ciencia Hoy 18:8-17.

BRANQUART, E., K. VERHEYEN \& J. LATHAM. 2008. Selection criteria of protected forest areas in Europe: The theory and the real world. Biol. Conserv. 141: 2795-2806. https://doi.org/10.1016/j.biocon.2008.08.015

BRAUN-BLANQUET, J. 1979. Fitosociología. Bases para el estudio de las comunidades vegetales [ed. 3]. $\mathrm{H}$. Blume Ediciones, Barcelona.

BROWN, A., U. MARTINEZ ORTIZ, M. ACERBI \& J. CORCUERA. 2006. La Situación Ambiental Argentina 2005. Fundación Vida Silvestre Argentina, Buenos Aires.

BURKHARD, B., I. PETROSILLOAN \& R. COSTANZA. 2010. Ecosystem services-bridging ecology, economy and social sciences. Ecol. Complexity 7: 257-259.

https://doi.org/10.1016/j.ecocom.2010.07.001

CADENASSO, M.L., S.T.A. PICKETT \& K. SCHWARZ. 2007. Spatial heterogeneity in urban ecosystems: reconceptualizing land cover and a framework for classification. Front. Ecol. Environ. 5: 80-88.

h t t p s : // d o i.org/10.1890/15409295(2007)5[80:SHIUER]2.0.CO;2
CAIN, S. 1938. The species-area curve. The American Midland Naturalist 19: 573-581. http://dx.doi.org/10.2307/2420468

COSTANZA, R., R. D'ARGE, R. DE GROOT, S. FARBER, M. GRASSO, M., B. HANNON, LIMBURG, K., S. NAEEM, R.V. O'NEILL, J. PARUELO, R.G. RASKIN, P. SUTTON \& M. VAN DEN BELT. 1997. The value of the world's ecosystem services and natural capital. Nature 387: 253-259.

https://doi.org/10.1038/387253a0

DE GROOT, R.S. 2006. Function-analysis and valuation as a tool to assess land use conflicts inplanning for sustainable, multi-functional landscapes. Landsc. Urban Plan. 75:175-186.

https://doi.org/10.1016/j.landurbplan.2005.02.016

DIRZO, R. \& P. RAVEN. 2003. Global state of biodiversity and loss. Annu. Rev. Environ. Resour. 28: 137-167. https://doi org/10.1146/annurev. energy.28.050302.105532

DONALD, P. F. \& A. D. EVANS. 2006. Habitat connectivity and matrix restoration. J. Appl. Ecol. 43:209-218. https://doi.org/10.1111/j.1365-2664.2006.01146.x

ELOSEGI, A. \& S. SABATER. 2009. Conceptos y técnicas en ecología fluvial. Fundación BBVA, 1 ed.

ESCOBEDO, F.J., T. KROEGER \& J.E.WAGNER. 2011. Urban forests and pollution mitigation: Analyzing ecosystem services and disservices. Environ. Pollut. 159: 2078-2087. https://doi.org/10.1016/j.envpol.2011.01.010

FARLEY, J. \& R. COSTANZA. 2010. Payments for ecosystem services: From local to global. Ecol. Econ. 69: 2060-2068. https://doi.org/10.1016/j.ecolecon.2010.06.010

FERNÁNDEZ, L., J. RAU \& A. ARRIAGADA. 2009. Calidad de la vegetación ribereña del río Maullín (4128'S;7259'W) utilizando el índice QBR. Gayana Bot. 66: 269-278. http://dx.doi.org/10.4067/S071766432009000200011

FORMAN, R.T.T. (ed) (1995). Land Mosaic: The Ecology of Landscapes and Regions. Great Britain: Cambridge University Press.

FRANK, F., C. FÜRST, L. KOSCHKE \& F. MAKESCHIN. 2012. A contribution towards a transfer of the ecosystem service concept to landscape planning using landscape metrics. Ecol. Indic. 21: 30-38. https://doi.org/10.1016/j.ecolind.2011.04.027

FULLER, R.A., K.N. IRVINE, P. DEVINE-WRIGHT, P.H WARREN \& K.J. GASTON. 2007. Psychological benefits of green space increase with biodiversity. Biol. Lett. 3: 390-394. https://doi.org/10.1098/rsbl.2007.0149

GALLARDO, B., S. GASCÓN, X. QUINTANA \& F.A. COMÍN. 2011. How to choose a biodiversity indicator - Redundancy and complementarity of biodiversity metrics in a freshwater ecosystem. Ecol. Indic. 11: 11771184. https://doi.org/10.1016/j.ecolind.2010.12.019 
GÓMEZ OREA, D. \& A. GÓMEZ VILLARINO. 2013. Ordenación Territorial. Madrid Mundi: Prensa.

GONZÁlEZ DEL TÁNAGO, M., D. GARCÍA DE JALÓN, F. LARA \& R. GARILLETI. 2006. Índice RQI para la valoración de las riberas fluviales en el contexto de la directiva marco del agua. Ingeniería Civil 143: 97-108.

GORDON A., D. SIMONDSON, M. WHITE, A. MOILANENC \& S.A. BEKESSY. 2009. Integrating conservation planning and land use planning in urban landscapes. Landsc. Urban Plan. 91: 183194.

https://doi.org/10.1016/j.landurbplan.2008.12.011

GRAU, H. R., N. I. GASPARRI \& T. M. AIDE. 2005. Agriculture expansion and deforestation in seasonally dry forests of northwest Argentina. Environ. Conserv. 32: 140-148. https://doi.org/10.1017/S0376892905002092

GUTIÉRREZ, C., A. SALVAT \& F. SABATER. 2001. Índex per a l'avaluació de la qualitat del medi fluvial a partir de la vegetació de ribera, Index IVF. Documents Tecnics del'Agencia Catalana de l'Aigua.

JAEGER, J. A.G., R. BERTILLER, C. SCHWICK \& F. KIENAST. 2010. Suitability criteria for measures of urban sprawl. Ecol. Indic. 10: 397-406. https://doi.org/10.1016/j.ecolind.2009.07.007

KAIRO, M., B. ALI, O. CHEESMAN, K. HAYSOM \& S. MURPHY. 2003. Invasive Species Threats in the Caribbean Region. Report to The Nature Conservancy. CAB international.

KANDUS, P., D.R. QUINTANA. R.F. BÓ (eds). 2006. Patrones de paisaje y biodiversidad del bajo delta del río Paraná. Mapa de ambientes. 1a ed. - Buenos Aires: Pablo Casamajor.

LAWSON, D.M., C.K. LAMAR \& M.W., SCHWARTZ. 2008. Quantifying plant population persistence in human-dominated landscapes. Conserv. Biol. 22: 922-928. https://doi.org/10.1111/j.1523-1739.2008.00936.x

LI F, R. WANG, J. PAULUSSEN \& X. LIU. 2005. Comprehensive concept planning of urban greening based on ecological principles: A case study in Beijing, China. Landsc. Urban Plan. 72: 325-336. https://doi.org/10.1016/j.landurbplan.2004.04.002

LIN Y., N. HONGB, P. WU, C. WU \& P. H. VERBURG. 2007. Impacts of land use change scenarios on hydrology and land use patterns in the Wu-Tu watershed in Northern Taiwan. Landsc. Urban Plan. 80: 111-126. https://doi.org/10.1016/j.landurbplan.2006.06.007

LINDENMAYER, D., R.J. HOBBS, R. MONTAGUEDRAKE \& E. ZAVALETA. 2008. A checklist for ecological management of landscapes for conservation. Ecol. Lett. 11: 78-91. https://doi.org/10.1111/j.1461-0248.2007.01114.X
MAGDALENO, F., R. MARTÍNEZ \& V. ROCH. 2010. Índice RFV para la valoración del estado del bosque de ribera. Ingeniera Civil 157: 85-96.

MINISTERIO DE AMBIENTE Y DESARROLLO SUSTENTABLE DE LA NACIÓN. 2016. Estrategia Nacional sobre Biodiversidad. Plan de Acción 2016-2020. [Online]. Disponible en: https://www.argentina.gob.ar/sites/default/files/ estrategia-biodiversidad_2016-2020.pdf [Acceso: 20 de junio de 2018].

MUNNÉ, A., C. SOLÁ \& N. PRAT. 1998. QBR: Un índice rápido para la evaluación de la calidad de los ecosistemas de ribera. Tecnología del agua 175: 20-37.

NATALE, E., G. A. MARTINEZ, A. J. OGGERO, M. D. ARANA, G. VILlalBA \& H. REINOSO. 2014. Evaluación del estado de conservación de remanentes naturales y seminaturales en la cuenca del rio Chocancharava (Córdoba, Argentina) a través de índices de calidad de hábitat. XI Congreso Latinoamericano de Botánica. Salvador de Bahía, Brasil. [Online]. Disponible en: http://www.botanica.org.br/trabalhoscientificos/65CNBot/5116-FLF.pdf [Acceso: 20 junio 2018].

NATALE, E., G. VILLALBA, J. E. JUNQUERA \& S. M. ZALBA. 2015. Assessment of the conservation status of natural and semi-natural patches associated with urban areas through habitat suitability indices. Int. J. Environ. Res. 9: 495-504. https://doi.org/10.22059/IJER.2015.923

OGGERO, A. J., N. DE LUCA, E. NATALE \& M. D. ARANA. 2014. Caracterización y situación actual de los bosques nativos en el centro sur de la provincia de Córdoba. Voces en el Fénix 142-151.

PARCERISAS, L., J. MARULL, J. PINO, E. TELLO, F. COLL \& C. BASNOU. 2012. Land use changes, landscape ecology and their socioeconomic driving forces in the Spanish Mediterranean coast (El Maresme County, 1850-2005). Environ. Sci. Policy 23: $120-130$.

https://doi.org/10.1016/j.envsci.2012.08.002

PRIMACK, R. \& J. ROS. 2000. Introducción a la Biología de la Conservación. $1^{\circ}$ edición. Ariel, España.

ROMERO, F. I., M. A. COZANO, R. A. GANGAS \& P. I. NAULIN. 2014. Zonas ribereñas: protección, restauración y contexto legal en Chile. Bosque 35: $3-12$. http://dx.doi.org/10.4067/S0717-92002014000100001

SAGRIPANTI, G., J. CONIGLIO \& A. CABRERA, (Autores) E. MATTEODA, N. DOFFO \& G. VILLALBA(colaboradores). 2005. Hoja Geológica 3363-III, Río Cuarto, Provincia de Córdoba. Instituto de Geología y Recursos Minerales, SEGEMAR. Boletín 349. Buenos Aires. 


\section{E. Natale et al. - Caracterización ambiental de la cuenca del río Cuarto}

SANDSTRÖM, U. G., P. ANGELSTAM \& A. KHAKEE. 2006. Urban comprehensive planning - identifying barriers for the maintenance of functional habitat networks. Landsc. Urban Plan. 75: 43-57. https://doi.org/10.1016/j.landurbplan.2004.11.016

STOCKER, T. F., D. QIN, G. PLATTNER, M. M. TIGNOR, M. SIMON, K. ALLEN, J. BOSCHUNG, A. NAUELS, Y. XIA, V. BEX \& P. M. MIDGLEY. 2013. Cambio Climático. Bases Físicas. Contribución del grupo de trabajo y al quinto informe de evaluación del grupo intergubernamental de expertos sobre el cambio climático. Grupo Intergubernamental de Expertos sobre el Cambio.

SZPEINER, A., M. A. MARTINEZ-GHERSA \& C. GHERSA. 2007. Agricultura pampeana, corredores biológicos y biodiversidad. Ciencia Hoy 101: 3846.

THIERS, B. [continuously updated]. 2018. Index Herbariorum: a global directory of public herbaria and associated staff. New York Botanical Garden's Virtual Herbarium. [Online]. Disponible en: http:// sweetgum.nybg.org/ih [Acceso: 5 octubre 2017].

UNIDAD DE MANEJO DEL SISTEMA DE EVALUACIÓN FORESTAL (UMSEF). 2015. Monitoreo de la superficie de bosque nativo de la República Argentina. Periodo 2013-2014. Secretaría de ambiente y desarrollo sustentable de la Nación. 67pp.

UUEMAA, E., M. ANTROP, J. ROOSAARE, R. MARJA \& U. MANDER. 2009. Landscape Metrics and Indices: An Overview of Their Use in Landscape Research. Living Review Landscape Research 3. [Online]. Disponible en: http://www.livingreviews. org/lrlr-2009-1. [Acceso: 7 de noviembre de 2015]

VAN DER BIEST, K., R. D'HONDT, S. JACOBS, D. LANDUYT, J. STAES, P. GOETHALS \& P. MEIRE. 2014. EBI: An index for delivery of ecosystem service bundles. Ecol. Indic. 37: 252265.

https://doi.org/10.1016/j.ecolind.2013.04.006

WILLIAMS N. S. G., M. V. SCHWARTZ, P. A. VESK, M. A. MCCARTHY, A. K. HAHS, S. E. CLEMANTS, R. T. CORLETT, R. P. DUNCAN, B. A. NORTON, K. THOMPSON \& M. J. MCDONNELL. 2009. A conceptual framework for predicting the effects of urban environments on floras. J. Ecol. 97: 4-9. https://doi.org/10.1111/j.1365-2745.2008.01460.x

ZAK, M., M. CABIDO, D. CÁCERES \& S. DÍAZ. 2008. What drives accelerated land cover change in central Argentina?. Enviro. Manage. 42: 181-189. https://doi.org/10.1007/s00267-008-9101-y 


\section{E. Natale et al. - Caracterización ambiental de la cuenca del río Cuarto}

\section{Anexo I}

\section{Descripción del indice integral de evaluación ambiental (IIEA) (Natale et al., 2015)}

Dicho índice está compuesto por tres componentes, la integridad ecológica conformada por las métricas del paisaje: tamaño (Ar), forma (Fr) y tipo de parche (Tr), tipo de contacto (Tc) y Funcionalidad ecológica (Fe) (Tabla 3), la importancia geológica dado por un valor de protección (Vp) y un valor de singularidad (Vs) ( para este estudio definidas en la sección resultados), y por variables de contexto referentes al impacto antrópico que incluye la presencia de caminos $(\mathrm{Pc})$, presencia de asentamientos $(\mathrm{Pa})$, porcentaje de superficie raleada (R) y/o desmontada (D) y porcentaje de superficie invadida (Pi). Las variables definidas en cada uno de estos componentes se integran en una ecuación general que, mediante algoritmos matemáticos, intentan reflejar la interacción de las mismas (Ecuación 1).

Tabla 3. Definición de las variables de integridad ecológica.

\begin{tabular}{|c|c|c|}
\hline Variables & Estados & Definición \\
\hline \multirow[t]{5}{*}{ Tipo de parche } & Disturbio & Resulta de la alteración o disturbio de un área pequeña \\
\hline & Remanente & $\begin{array}{l}\text { Aparece como una pequeña área que escapó a algún disturbio } \\
\text { de los alrededores }\end{array}$ \\
\hline & Ambiental & Es causada por las condiciones naturales del ambiente físico \\
\hline & Regeneración & $\begin{array}{l}\text { Aparece cuando la vegetación se ha recuperado después de } \\
\text { un disturbio. }\end{array}$ \\
\hline & Introducido & $\begin{array}{l}\text { Generado por la acción del hombre a través de plantación de } \\
\text { especies exóticas (ornamentales principalmente) }\end{array}$ \\
\hline \multirow[t]{3}{*}{ Forma del parche } & Regular & $\begin{array}{l}\text { Sin bordes intrincados y donde las dimensiones principales } \\
\text { tienen una relación menor a } 2: 1 \text { (Puede ser natural o antrópico } \\
\text { que se distinguen por la forma de los bordes; limites naturales } \\
\text { son curvados mientras que los creados por el hombre tienen } \\
\text { una o más líneas rectas) }\end{array}$ \\
\hline & Irregular & $\begin{array}{l}\text { Cantidad de lóbulos principales presentes, probablemente } \\
\text { como resultado de la heterogeneidad espacial o temporal en } \\
\text { los proceso de control. (Puede ser natural o antrópico que se } \\
\text { distinguen por la forma de los bordes) }\end{array}$ \\
\hline & Alargado & $\begin{array}{l}\text { Se mide por la relación largo-ancho con una relación mayor a } \\
2: 1 \text { sin bordes intrincados. (Puede ser natural o antrópico que } \\
\text { se distinguen por la forma de los bordes) }\end{array}$ \\
\hline \multirow[t]{4}{*}{ Tipo de contacto } & Florístico natural & Contacto gradual entre dos remanentes de vegetación nativa \\
\hline & Florístico introducido & $\begin{array}{l}\text { Contacto de un remanente de vegetación natural con } \\
\text { remanentes introducido }\end{array}$ \\
\hline & Estructural Natural & $\begin{array}{l}\text { Contacto entre dos remanentes de vegetación natural pero } \\
\text { en distintas etapas sucesionales (Después de un disturbio } \\
\text { antrópico) }\end{array}$ \\
\hline & Estructural Antrópico & $\begin{array}{l}\text { Contacto de un remanente natural con sitios con disturbio } \\
\text { antrópico (desmonte, raleo, fuego, etc.) }\end{array}$ \\
\hline \multirow{2}{*}{$\begin{array}{l}\text { Funcionalidad } \\
\text { ecológica }\end{array}$} & Corredo & Tipo de parche alargado \\
\hline & No corredor & \\
\hline
\end{tabular}


A cada variable se le asignó un valor de aptitud de índice que va de 0 a 1 donde 0 es la peor condición y 1 la condición más adecuada (Fig. 11; 12 y Tabla 4).

$$
\begin{gathered}
\left.(\mathrm{IIEA})=\left[\left(((\mathrm{Pc}+\mathrm{Pa}+\mathrm{D}+\mathrm{R}) / 4)^{*} \mathrm{Pi}\right)^{2 *} *((\mathrm{Fr}+\mathrm{Tr}+\mathrm{Tc}) / 3) * \mathrm{Ar}\right)^{2 *}((\mathrm{Vp}+\mathrm{Vs}) / 2)\right]^{5}+[(((\mathrm{Pc} \\
\left.\left.+\mathrm{Pa}+\mathrm{D}+\mathrm{R}) / 4) * \mathrm{Pi})^{2 *}(((\mathrm{Fr}+\mathrm{Tr}+\mathrm{Tc}) / 3) * \mathrm{Ar})^{2 *}((\mathrm{Vp}+\mathrm{Vs}) / 2)\right)\right]^{5 * 0,1 * \mathrm{Fe})}(1)
\end{gathered}
$$
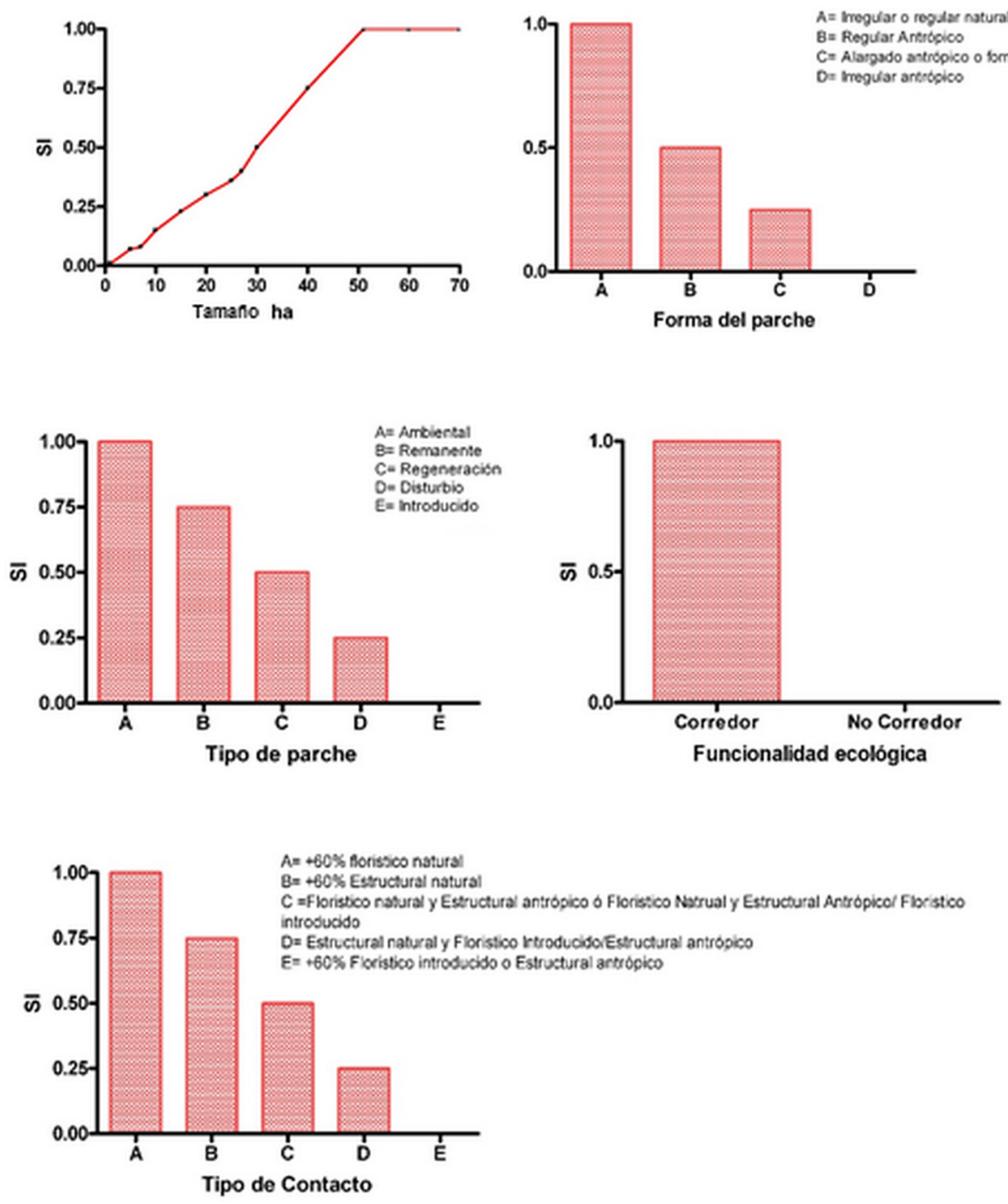

Fig. 11. Valores de aptitud de índice (SI) por variable de integridad ecológica. 


\section{E. Natale et al. - Caracterización ambiental de la cuenca del río Cuarto}
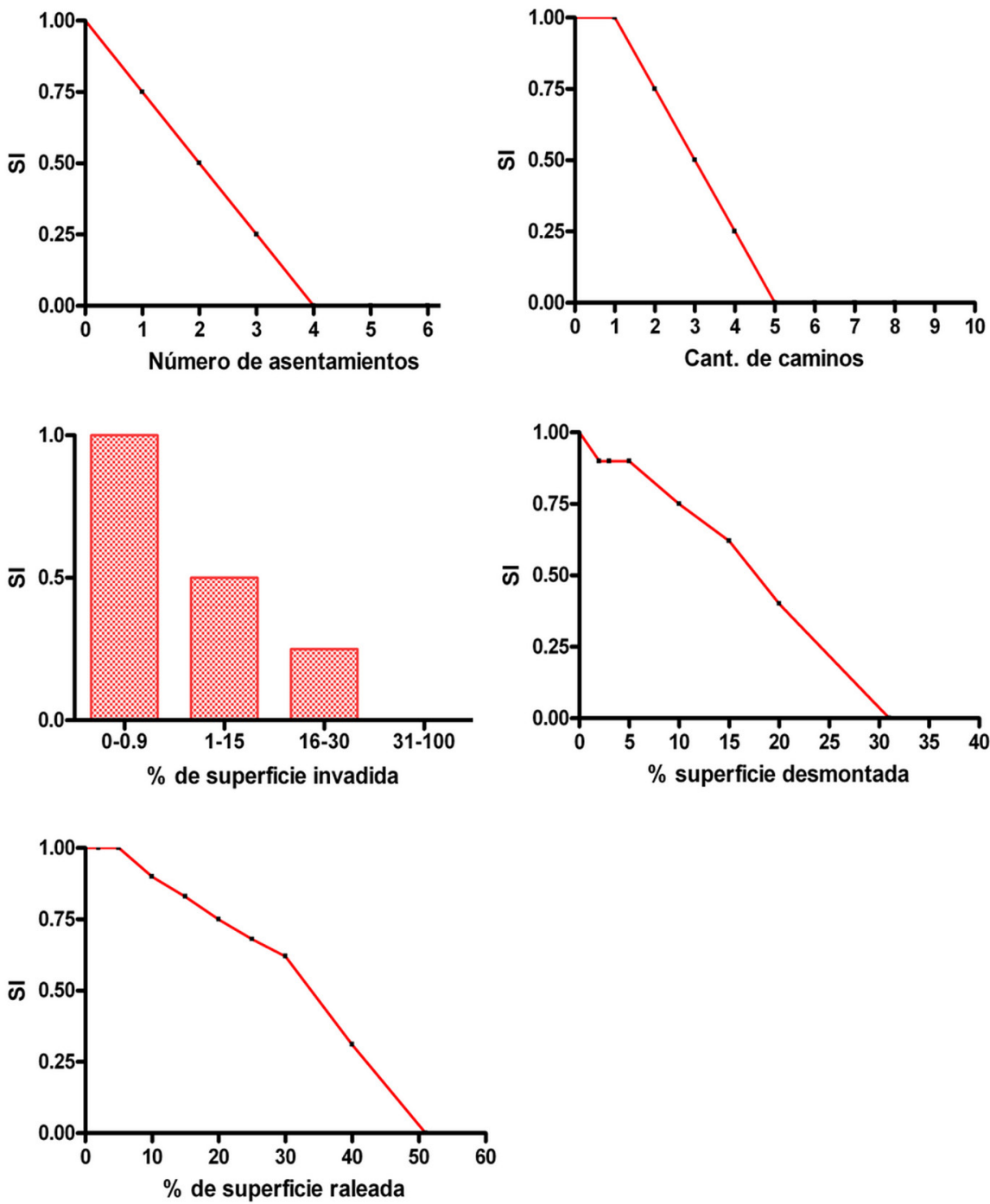

Fig. 12. Valores de aptitud de índice (SI) para variable de impacto antrópico.

Tabla 4. Asignación de aptitud de índice para las variables del componente geológico.

\begin{tabular}{|llll|}
\hline \multicolumn{1}{|c}{ Variable } & \multicolumn{1}{c|}{ Alto (1) } & \multicolumn{1}{c|}{ Medio $(\mathbf{0 , 5 )}$} & \multicolumn{1}{c|}{ Bajo $(\mathbf{0 , 2 )}$} \\
Valor de protección & $\begin{array}{l}\text { 2 o más variables con valores } \\
\text { altos de susceptibilidad }\end{array}$ & $\begin{array}{l}\text { Dominio de valores medios } \\
\text { de susceptibilidad o uno } \\
\text { de cada categoría }\end{array}$ & $\begin{array}{l}\text { 2 o más variables } \\
\text { con valores bajos de } \\
\text { susceptibilidad }\end{array}$ \\
\hline
\end{tabular}

